Cahiers Charlevoix

Études franco-ontariennes
Cahiers Charlevoix Études franco-ontariennes

or Crevenerix of

\title{
Les perspectives amoureuses et conjugales chez les jeunes du nord-est de l'Ontario
}

\section{Simon Laflamme}

Volume 10, 2014

URI : https://id.erudit.org/iderudit/1039292ar

DOI : https://doi.org/10.7202/1039292ar

Aller au sommaire du numéro

\section{Éditeur(s)}

Société Charlevoix

Presses de l’Université d’Ottawa

ISSN

1203-4371 (imprimé)

2371-6878 (numérique)

Découvrir la revue

Citer cet article

Laflamme, S. (2014). Les perspectives amoureuses et conjugales chez les jeunes du nord-est de l'Ontario. Cahiers Charlevoix, 10, 171-209.

https://doi.org/10.7202/1039292ar
Résumé de l'article

Simon Laflamme poursuit ici sa comparaison entre les Ontariens, selon qu'ils sont anglophones ou francophones, et fait état de quelques différences entre les populations, mais surtout d'une grande similitude. La comparaison porte cette fois sur les perspectives amoureuses et familiales des jeunes du nord-est de l'Ontario. On pourrait s'imaginer que, compte tenu des statistiques de la vie conjugale des dernières décennies, lesquelles témoignent d'abondantes ruptures, ces perspectives soient plutôt pessimistes. L'étude montre que ce n'est pas tout à fait le cas : s'il y a quelques doutes, l'avenir conjugal et parental est, dans l'ensemble, envisagé avec optimisme. Et, malgré quelques particularismes, ce constat se vérifie aussi bien chez les francophones que chez les anglophones. Pour tenter d'expliquer autrement les représentations, l'auteur a aussi fait intervenir quelques facteurs additionnels, selon que le jeune se trouve au début ou à la fin des études secondaires, selon la taille de la communauté de résidence, le caractère religieux ou non de l'école, et le genre. Il a souvent trouvé des différences, mais jamais telles qu'elles obligent à constater de grands écarts. Les problématiques de l'amour et de la famille, dans l'esprit des jeunes, comportent des spécificités, mais elles sont surtout marquées du sceau de la transcendance.
Ce document est protégé par la loi sur le droit d'auteur. L'utilisation des services d'Érudit (y compris la reproduction) est assujettie à sa politique d'utilisation que vous pouvez consulter en ligne.

https://apropos.erudit.org/fr/usagers/politique-dutilisation/ 


\title{
Les perspectives amoureuses et conjugales chez les jeunes du nord-est de l'Ontario
}

\author{
Simon LAFLAMme \\ Département de sociologie \\ Université Laurentienne
}




\section{SOMMAIRE}

1. INTRODUCTION 173

2. MÉThode 178

3. De faibles VARIations ET Des Similitudes 180

3.1. Le genre 180

3.2. L'année d'études 184

3.3. La taille de la communauté de résidence 186

3.4. La langue 188

3.5. La religion 193

3.6. L'interaction des facteurs 195

3.7. Des opinions plutôt nettes, des nuances et des propos généralisés 203

4. Conclusion 205 


\section{Les perspectives amoureuses et conjugales chez les jeunes du nord-est de l'Ontario ${ }^{1}$}

\section{INTRODUCTION}

Dans les précédents numéros des Cahiers Charlevoix, nous avons régulièrement mis en parallèle les francophones et les anglophones de l'Ontario. Dans le sixième cahier, la comparaison portait sur les médias qu'on trouve dans les foyers et sur l'usage qu'on en fait $^{2}$. Le septième poursuivait cette analyse en la centrant sur les usages et les représentations d'Internet ${ }^{3}$. Dans le huitième, le rapprochement avait pour objet les aspirations éducationnelles et professionnelles des jeunes ${ }^{4}$. Avec le neuvième, enfin, l'étude se transportait sur le thème des conditions sociales et des représentations qui entrent en jeu dans la transition que doivent faire les élèves du programme élémentaire vers le secondaire ${ }^{5}$. Tous ces travaux ont mis en évidence de grandes similitudes entre les francophones et les anglophones, mais tous ont également fait état de particularismes. Nous avons interprété cette dualité comme la manifestation d'une dialectique de l'homogénéisation et de la différenciation et n'avons pas manqué d'inscrire cette tension dans

1. Les analyses que comporte ce texte n'auraient pas été possibles sans le soutien de la Commission de formation du Nord-Est.

2. Simon Laflamme, "Les Médias en Ontario chez les francophones et les anglophones ", Cahiers Charlevoix, Sudbury, Société Charlevoix / Prise de parole, $\mathrm{n}^{\circ}$ 6, 2004, p. 251-282.

3. Simon Laflamme, «Usage et représentation d'Internet chez les jeunes. Comparaison entre les francophones et les anglophones du nord de l'Ontario ", Cahiers Charlevoix, Sudbury, Société Charlevoix / Prise de parole, nº 7, 2006, p. 183-228.

4. Simon Laflamme, «Le Nouveau Visage de l'ambition en Ontario français. Le cas du Nord-Est », Cahiers Charlevoix, Ottawa, Société Charlevoix / Presses de l'Université d'Ottawa, no 8, 2010, p. 77-110.

5. Simon Laflamme, «Passage de l'élémentaire au secondaire et décrochage culturel en Ontario français. Élimination de quelques facteurs », Cahiers Charlevoix, Ottawa, Société Charlevoix / Presses de l'Université d'Ottawa, nº 9, 2012, p. 15-59. 
la postmodernité, ce qui a permis d'en relever la spécificité en ce qui concerne l'Ontario anglophone et francophone. Ces travaux en tête, on peut se demander comment réapparaîtrait le dualisme dans une analyse de phénomènes qui font davantage appel à l'émotion, comme, par exemple, les perspectives amoureuses et familiales des jeunes.

Dans les pays industrialisés, la probabilité qu'un mariage se solde en un divorce est élevée. Le Canada ne fait pas exception. On y calcule, là, l'indice synthétique de divortialité ${ }^{6}$ qu'on présente communément en vertu de trente années ; or, depuis la fin des années 1980, la proportion oscille entre $35 \%$ et $42 \%$; il s'agit du risque qu'un mariage se termine par un divorce avant que les époux n'aient pu en fêter le trentième anniversaire. Ces statistiques de désunion s'accentueraient sans doute quelque peu si elles prenaient en considération « les séparations de corps, les divorces accordés dans d'autres pays et l'abandon du domicile conjugal $^{7} \gg$. Elles augmenteraient davantage si étaient comptabilisées les ruptures qui impliquent des conjoints de fait, compte tenu que la proportion des familles à l'intérieur desquelles on trouve un couple marié a diminué de 70,5\% à 67,0\% de 2001 à 2011 alors que celle des unions libres, elle, a crû de 13,8\% à $16,7 \%$ pendant la même période ${ }^{8}$ et que la probabilité d'une séparation est plus forte dans le cas des unions libres que dans

6. «L'indice synthétique de divortialité est un indicateur qui permet de décrire l'intensité de la divortialité en présentant la proportion de mariages qui, pour 1000 mariages au départ, se termineraient par un divorce si les taux de divortialité, calculés par durée de mariage une année civile donnée, s'appliquaient à ces 1000 mariages. En termes mathématiques concrets, l'indice synthétique de divortialité correspond à la somme des taux de divortialité par durée de mariage » (Ressources humaines et développement des compétences Canada, "Glossaire - Divorce », Indicateurs de mieuxêtre au Canada, http://www4.hrsdc.gc.ca/gl.4ss.1ry@-fra.jsp?word=Divorce\#indice synthétique de divortialité, site consulté en février 2013).

7. L'Encyclopédie canadienne, "Mariage et divorce ", Historica-Dominion, http://www.thecanadianencyclopedia.com/articles/fr/mariage-et-divorce (site consulté en février 2013).

8. Statistique Canada, «Recensement de la population de 2011 : familles, ménages, état matrimonial, type de construction résidentielle, logements collectifs », Le Quotidien, Gouvernement du Canada, 19 septembre 2012, http://www.statcan.gc.ca/ daily-quotidien/120919/dq120919a-fra.htm (site consulté en février 2013). 
celui des mariages ${ }^{9}$. On peut greffer à ces données que les unions libres sont moins durables que ne le sont les mariages ${ }^{10}$. Si l'on spécifie que l'âge moyen du divorce se situe au début de la quarantaine ${ }^{11}$ et que celui de la séparation pour les couples non mariés est souvent inférieur, on peut en déduire que bon nombre d'enfants éprouvent la séparation de leurs parents et que, s'ils n'ont pas assisté à celle de leurs propres parents, ils ont sans doute témoigné de celle des parents de leurs camarades. La fragilité des relations amoureuses fait concrètement partie de leur vécu. On peut donc se demander si l'adolescent n'envisage pas avec pessimisme la vie à deux.

Cette question est rarement abordée de front; non pas par négligence, mais plutôt parce que les spécialistes de la famille ont beaucoup à faire avec d'autres thématiques, comme les relations entre les conjoints, entre les parents et les enfants ou entre les enfants eux-mêmes, et, corrélativement, comme l'économie domestique ; ils sont beaucoup interpellés en plus par des sujets comme la diversité des structures et l'infertilité. Et toutes les études qu'ils produisent sont bien au fait de la précarité des relations conjugales.

Des auteurs célèbres ont fait état des grandes attentes des partenaires dans les couples de la postmodernité, et donc de la vulnérabilité de leur relation ${ }^{12}$. Si la modernité a diversifié

9. «Bien que les couples mariés soient beaucoup plus nombreux au Canada que les couples en union libre [...], les données reflètent la plus grande probabilité de rupture des unions libres comparée à celle des mariages [...]» (Statistique Canada, Section 2 : vivre une rupture d'union, http://www.statcan.gc.ca/pub/89-625-x/ 2007002/4055019-fra.htm\#1, site consulté en février 2013).

10. « [L]'étude a montré que, chez les couples ayant récemment mis fin à leur union, ceux qui étaient en union libre avaient vécu ensemble, en moyenne, 4,3 années, soit 10 ans de moins que les 14,3 années, en moyenne, chez les gens mariés » (Statistique Canada, Section 2 : vivre une rupture d'union, http://www.statcan.gc.ca/pub/ 89-625-x/2007002/4055019-fra.htm\#1, site consulté en février 2013).

11. En 2008, cet âge moyen est de 41,9 ans pour les femmes et de 44,5 pour les hommes (Ressources humaines et développement des compétences Canada, «Vie familiale - Divorce ", Indicateurs de mieux-être au Canada, http://www4.hrsdc. gc.ca/.3ndic.1t.4r@-fra.jsp?iid=76, site consulté en février 2013).

12. Antony Giddens, La Transformation de l'intimité. Sexualité, amour et érotisme danslessociétésmodernes,Paris,LeRouergue/Chambon,[1992]2004;NiklasLuhmann, Amour comme passion. De la codification de l'intimité, Paris, Aubier, [1982] 1990. 
la composition des sociétés en référence aux communautés antérieures, la postmodernité n'a fait qu'amplifier le processus. Par voie de conséquence, elle a intensifié la probabilité que les conjoints aient des origines différentes. Cette altérité ne peut pas ne pas se répercuter dans un milieu familial. Tout un numéro de la revue Enfances, familles, générations a été consacré au phénomène sous le titre Regards croisés sur la mixité conjugale ${ }^{13}$. Tous les articles invitent à un renouveau de la conceptualisation des relations conjugales pour appréhender ces unions et leur environnement. Avec la postmodernité, s'est aussi complexifiée la gestion du temps : les parents sont tiraillés entre les exigences du travail et celles de la famille, et les points d'équilibre sont plutôt instables, comme le soulignent Annette Tézli et Anne H. Gauthier ${ }^{14}$. Et cet équilibre fait l'objet de réflexions : aux yeux de Joseph G. Grzywacz et Dawn S. Carlson, il se dessine dans les négociations entre les conjoints des attentes relatives aux rôles familiaux et professionnels ${ }^{15}$.

La manière dont circule l'argent dans un milieu familial fait aussi objet d'attention parce que lui sont associées la question des positions des membres dans la structure et celle de leurs rôles. Hélène Belleau et Raphaëlle Proulx y répondent en mettant en évidence le pouvoir des jeunes sur les parents ${ }^{16}$. Peter Burton et Shelley Phipps indiquent que, au Canada, le revenu familial tend à stagner, surtout au sein des classes moyennes et ils invitent à parler davantage d'inégalité de bien-être que d'inégalité de revenu ${ }^{17}$.

Les travaux qui portent sur la famille contemporaine

13. Josiane Le Gall et Catherine Therrien (dir.), n 17, automne 2012.

14. Annette Tézli et Anne H. Gauthier, «Balancing Work and Family in Canada : An Empirical Examination of Conceptualizations and Measurements », Canadian Journal of Sociology, vol. 34, n 2, 2009, p. 433-462.

15. Joseph G. Grzywacz et Dawn S. Carlson, « Conceptualizing Work Family Balance : Implications for practice and Research », Advances in Developing Human Resources, $\mathrm{n}^{\circ}$ 9, 2007, p. 455-471.

16. Hélène Belleau et Raphä̈lle Proulx, « Le Revenu familial, un concept vague et insidieux : analyse critique et historique des relations économiques familiales », Enfances, familles, générations, $\mathrm{n}^{\circ} 15$, automne 2011, p. 78-109.

17. Peter Burton et Shelley Phipps, «Families, Time, and Well-Being in Canada», Canadian Public Policy, vol. 37, n 3, 2011, p. 395-423. 
s'interrogent beaucoup sur ses nouvelles configurations. Martine Fournier, dans une perspective historique, décrit cette diversification des modèles familiaux et la relie à l'économie autant qu'à la démographie ${ }^{18}$. Anne Cadoret se penche sur l'homoparentalité et y voit une continuité par rapport à la parentalité traditionnelle en ce qu'il s'agit toujours de deux parents qui éduquent un enfant, mais note une rupture parce que les parents ne sont plus définissables comme géniteurs ; 1'auteur discute en outre du problème de la reconnaissance de ces familles en France ${ }^{19}$.

Quand les spécialistes de la famille abordent la question de l'infertilité, ils peuvent examiner le rapport entre les conjoints qui sont en quête d'enfant et les médecins qui leur donnent à espérer une procréation assistée, comme le fait Doris Chateauneuf qui découvre une dualité des discours ${ }^{20}$.

Des recherches s'intéressent à la vie amoureuse des jeunes, souvent à des fins éducatives ${ }^{21}$; mais il en est peu qui portent sur leurs aspirations affectives et familiales. L'objectif de ce texte que nous proposons est précisément d'examiner ces perspectives. Des questions précises se posent. Comment les jeunes envisagent-ils leurs relations amoureuses ? Comment entrevoient-ils les relations qu'ils auront avec leurs enfants ? Comment se présentera, à leurs yeux, la relation entre leur conjoint et les enfants?

Compte tenu de la persistance de schèmes traditionnels, on peut penser que les réponses à ces questions varieront selon le sexe, que les filles seront plus enclines à donner cours à des idéaux

18. Martine Fournier, «Famille : la question du modèle. Une approche par les courants historiques et sociologiques ", Informations sociales, $n^{\circ} 115,2004$, p. 0-69.

19. Anne Cadoret, « La Famille homoparentale en France : une révolution tranquille? », Enfances, familles, générations, n 15, automne 2011, p. 45-60.

20. Doris Phaneuf, «Projet familial, infertilité et désir d'enfant : usages et expériences de la procréation médicalement assistée en contexte québécois », Enfances, familles, générations, $\mathrm{n}^{\circ} 15$, automne 2011, p. 61-71.

21. Lorraine Rondeau et Chantal Hamel (dir.), Les Relations amoureuses des jeunes. Écouter pour mieux accompagner, Montréal, Direction de la santé publique, Agence de la santé et des services sociaux de Montréal, 2008, http://publications. santemontreal.qc.ca/uploads/tx_asssmpublications/978-2-89494-633-6.pdf (site consulté en mars 2013). 
conjugaux et familiaux. On peut aussi s'attendre à ce que, par effet de sagesse, les jeunes adultes de dix-sept ou dix-huit ans témoigneront d'une certaine circonspection dans leurs desseins alors que les adolescents de quatorze ou quinze ans, eux, imagineront plus volontiers un avenir heureux. Le fait qu'on vive en milieu rural ou urbain ne devrait pas avoir d'influence.

Par ailleurs, si l'on garde en tête les résultats que nous avons obtenus dans les études précédentes, on peut supposer que, à propos d'une réalité sociale aussi générale que celle de la famille, prenant en considération que les opinions seront influencées par un événement aussi commun que celui de la désunion, le fait, au Canada, d'être francophone ou anglophone, minoritaire ou majoritaire, ne devrait pas avoir d'incidence. On peut par contre concevoir que les projets de mariage puissent être déterminés par la religion, les catholiques se projetant davantage que les autres dans une union ainsi consacrée.

\section{MÉTHOde}

Pour répondre à ces questions, pour vérifier ces hypothèses, nous avons utilisé les données de la première année (2005) de 1'enquête Les Jeunes et le Nord : un parcours à découvrir ${ }^{22}$. Ces données ont été recueillies auprès des élèves de neuvième et de douzième années dans la plupart des écoles du Nord-Est de l'Ontario, région qui s'étend de Hearst au Témiskaming et qui va de Timmins à la Baie de James ${ }^{23}$. Plus de 1700 jeunes ont répondu à un questionnaire. Dans ce questionnaire, ils ont, entre autres, été invités à se prononcer à l'égard de quatorze énoncés sur le thème de la famille, des énoncés comme «ma vie amoureuse sera stable », " il vaut mieux avoir un emploi stable avant de vivre en couple », « il vaut mieux être marié-e avant d'avoir des

22. Simon Laflamme et Pierre Bouchard, Les Jeunes et le Nord. Un parcours à découvrir, Hearst, Commission de formation du Nord-Est, 2005, http://www. fnetbcfne.on.ca/pdf/Rapports/Les $\% 20$ jeunes $\% 20$ et $\% 201$ e $\% 20$ Nord $\% 20$ abrege $\% 20$ final.pdf.

23. Il s'agit, en fait, d'une sous-région du nord-est de la province de l'Ontario tel que ce Nord-Est est normalement défini administrativement. 
enfants » et « la relation entre moi et mon ou ma conjoint-e sera harmonieuse ». Il s'agit de projections, d'évocations de principes, d'idéaux. Chacune de ces propositions était attachée à une échelle à six niveaux dont les pôles étaient « pas du tout d'accord », soit 1, et « tout à fait d'accord», soit 6. Dans le questionnaire, les élèves ont indiqué leur sexe, leur année d'études, leur communauté de résidence et leur(s) langue(s) maternelle(s); on connaît l'école de chacun d'eux, ce qui permet de savoir si elle est de langue française ou anglaise de même que si elle appartient au système public ou catholique. Pour ce qui est de l'année d'études, elle se répartit en deux : neuvième et douzième ; pour ces deux groupes, l'âge moyen est respectivement de 14,5 et de 17,6 ans. Les communautés de résidence ont été ramenées à quatre groupements : 1) celles qui comptent moins de 1000 habitants, 2) celles qui comprennent entre 1000 et 4999 individus, 3) celles dont le nombre de citoyens oscille entre 5000 et 10999 et 4) la ville de Timmins (dans laquelle on dénombre plus de 40000 citadins). La variable langue maternelle a été catégorisée selon trois ensembles : 1) français, 2) anglais et 3) français et anglais ; les élèves qui appartiennent à d'autres catégories forment un assemblage marginal et ils ont été exclus des analyses. La langue de l'école comprend deux modalités : française et anglaise ; est aussi dichotomique l'indicateur de la religion qui se résume au caractère confessionnel ou non de l'établissement, les écoles étant ou bien catholiques, ou bien publiques. Les variables dépendantes, celles qui permettent d'observer, entre autres, la manière dont les jeunes se représentent la famille, sont construites sur des échelles de Likert (à six niveaux); il est donc permis de les traiter comme cardinales ${ }^{24}$. Les variables indépendantes peuvent être considérées comme nominales $^{25}$. Cela va de soi pour le sexe, la langue

24. Une variable est cardinale quand ses composants sont des valeurs numériques qui rendent possibles des opérations arithmétiques, comme le calcul de la moyenne. L'âge défini en années est une telle variable ; le revenu annuel en dollars et le poids en kilogrammes sont aussi des variables cardinales.

25. Une variable est nominale quand ses composants sont tout simplement des catégories. Le sexe est une variable nominale qui répartit les individus dans deux modalités (ou parfois plus). 
maternelle, la langue de l'école et la confessionnalité de l'école. On pourrait soutenir que les deux niveaux de scolarité forment une variable ordinale ${ }^{26}$, et non nominale; nous avons choisi de la traiter comme nominale parce qu'elle ne comporte que deux modalités et que notre intention est davantage de comparer des ensembles que de mesurer une association. On pourrait aussi avancer que la municipalité est de nature ordinale ; cependant, cette variable ne compte que quatre modalités et, à nouveau, c'est la comparaison entre les catégories qui nous intéresse ; nous n'avons donc pas hésité à l'employer comme nominale, et donc à la traiter comme les autres variables indépendantes. En procédant ainsi, nous avons pu effectuer des tests sur les comparaisons de moyennes entre les modalités de toutes les variables indépendantes et vérifier s'il fallait prendre en considération des effets d'interaction. Pour faciliter la présentation des résultats, nous avons fait porter les analyses sur chacun des facteurs, pris séparément, puis nous avons vérifié s'il fallait prendre en considération des effets interactifs de ces facteurs.

\section{DE FAIBLES VARIATIONS ET DES SIMILITUDES}

Dans cette section, nous rapportons les résultats de séries d'analyses, chacune se concentrant sur la relation entre un seul facteur et les quatorze objets sur lesquels les jeunes sont amenés à prendre position. Vient ensuite une description des analyses sur l'interaction des facteurs.

\subsection{Le genre}

La manière de concevoir la famille varie-t-elle selon le genre ?

Pour répondre à cette question, nous avons fait des tests $t$ (voir le tableau 1). Sur les quatorze énoncés, il y en a sept pour lesquels les moyennes des filles et des garçons doivent être considérées

26. Une variable est ordinale quand, entre ses composants, on peut reconnaître un ordre. Le niveau d'instruction est une variable ordinale. S'il comporte les modalités « élémentaire », " secondaire », « postsecondaire », alors non seulement y a-t-il une catégorisation, mais en plus on peut hiérarchiser ces catégories, postsecondaire étant supérieur à secondaire et secondaire étant supérieur à élémentaire. 
comme égales et sept où les différences ne doivent pas être prises comme un fruit du hasard.

Les jeunes, garçons ou filles, se prononcent de manière semblable devant la proposition qui veut que la vie à deux ne donne pas lieu au mariage ; les moyennes pour les deux ensembles sont inférieures à 2,50, elles expriment le désaccord, ce qui montre bien que le mariage fait communément partie des perspectives de la vie à deux. Filles et garçons sont aussi semblablement optimistes quand ils songent à la stabilité de leur vie amoureuse : les moyennes sont de 4,79 et 4,62 respectivement pour les unes et pour les autres. L'optimisme est encore plus élevé quand ils envisagent la relation entre l'éventuel conjoint et les enfants du couple ; cette relation sera harmonieuse : les moyennes sont proches du parfait assentiment : 5,37 pour les filles et 5,32 pour les garçons. Il n'y a pas non plus de différence entre les élèves de sexe féminin et ceux de sexe masculin quand ils évoquent le rapport entre la fin des études, d'une part, et la vie de couple ou le fait d'avoir des enfants d'autre part. Les jeunes des deux sexes tendent prudemment vers l'accord quand il est question de l'importance d'avoir terminé ses études avant de former un couple (les moyennes dépassant à peine la valeur de 4), mais l'acquiescement est plus marqué sur le principe de la fin des études comme préalable au statut de père ou de mère (les moyennes dépassant alors le seuil de 5). Les opinions sur le rapport entre l'emploi et la venue des enfants vont dans le même sens : garçons et filles croient fortement qu'il vaut mieux avoir d'abord un emploi stable (les moyennes sont à nouveau supérieures à 5). Le genre, enfin, n'a pas d'influence sur la façon de concevoir le lien entre le mariage et les enfants; qu'ils soient de sexe masculin ou féminin, les élèves inclinent prudemment à penser qu'il est préférable de faire précéder des épousailles l'arrivée des enfants (les moyennes franchissent la valeur de 4,50).

Filles et garçons présentent des moyennes inégales quand ils pensent au mariage : devant l'énoncé « plus tard je me marierai », la moyenne des premières est un peu plus élevée que celle des 
Tableau 1

Différence de moyennes pour diverses attitudes à l'égard de la famille selon le sexe de l'élève

\begin{tabular}{|c|c|c|c|c|c|c|}
\hline \multicolumn{7}{|c|}{ ( $1=$ pas du tout d'accord et $6=$ tout à fait d'accord $)$} \\
\hline \multirow[t]{2}{*}{ Énoncé } & & \multicolumn{2}{|c|}{ Sexe } & \multirow[t]{2}{*}{$\mathrm{t}$} & \multirow[t]{2}{*}{ ddl } & \multirow[t]{2}{*}{$\mathrm{p}<0,05$} \\
\hline & & Féminin & Masculin & & & \\
\hline \multirow[t]{2}{*}{ Plus tard, je me marierai } & $\overline{\mathrm{x}}$ & 5,06 & 4,79 & \multirow[t]{2}{*}{4,21} & \multirow[t]{2}{*}{1643,0} & \multirow[t]{2}{*}{ oui } \\
\hline & $\mathrm{S}$ & 1,31 & 1,34 & & & \\
\hline \multirow{2}{*}{$\begin{array}{l}\text { Plus tard, je vivrai avec } \\
\text { un-e conjoint-e, mais je } \\
\text { ne me marierai pas }\end{array}$} & $\overline{\mathrm{x}}$ & 2,40 & 2,28 & \multirow[t]{2}{*}{1,67} & \multirow[t]{2}{*}{1552} & \multirow[t]{2}{*}{ non } \\
\hline & $\mathrm{S}$ & 1,44 & 1,39 & & & \\
\hline \multirow{2}{*}{$\begin{array}{l}\text { Ma vie amoureuse } \\
\text { sera stable }\end{array}$} & $\overline{\mathrm{x}}$ & 4,79 & 4,62 & \multirow[t]{2}{*}{2,78} & \multirow[t]{2}{*}{1637} & \multirow[t]{2}{*}{ non } \\
\hline & $\mathrm{S}$ & 1,26 & 1,22 & & & \\
\hline \multirow{2}{*}{$\begin{array}{l}\text { Il vaut mieux avoir terminé } \\
\text { ses études avant de } \\
\text { vivre en couple }\end{array}$} & $\overline{\mathrm{x}}$ & 4,02 & 4,06 & \multirow[t]{2}{*}{$-0,58$} & \multirow[t]{2}{*}{1672,1} & \multirow[t]{2}{*}{ non } \\
\hline & $\mathrm{S}$ & 1,63 & 1,53 & & & \\
\hline \multirow{2}{*}{$\begin{array}{l}\text { Il vaut mieux avoir terminé } \\
\text { ses études avant d'avoir } \\
\text { des enfants }\end{array}$} & $\overline{\mathrm{x}}$ & 5,31 & 5,17 & \multirow[t]{2}{*}{2,46} & \multirow[t]{2}{*}{1686} & \multirow[t]{2}{*}{ non } \\
\hline & $\mathrm{S}$ & 1,19 & 1,22 & & & \\
\hline \multirow{2}{*}{$\begin{array}{l}\text { Il vaut mieux avoir un } \\
\text { emploi stable avant } \\
\text { de vivre en couple }\end{array}$} & $\overline{\mathrm{x}}$ & 3,99 & 4,42 & \multirow[t]{2}{*}{$-6,10$} & \multirow[t]{2}{*}{1650,4} & \multirow[t]{2}{*}{ oui } \\
\hline & $\mathrm{S}$ & 1,53 & 1,37 & & & \\
\hline Il vaut mieux avoir un & $\overline{\mathrm{x}}$ & 5,32 & 5,34 & $-0,43$ & 1671 & non \\
\hline $\begin{array}{l}\text { emploi stable avant } \\
\text { d'avoir des enfants }\end{array}$ & $\mathrm{S}$ & 1,09 & 1,06 & & & \\
\hline La relation entre moi et mes & $\overline{\mathrm{x}}$ & 5,50 & 5,41 & 2,00 & 1611 & oui \\
\hline enfants sera harmonieuse & $\mathrm{S}$ & 0,89 & 0,91 & & & \\
\hline La relation entre mon ou ma & $\overline{\mathrm{x}}$ & 5,37 & 5,32 & 1,03 & 1586 & non \\
\hline $\begin{array}{l}\text { conjoint-e et nos enfants sera } \\
\text { harmonieuse }\end{array}$ & $\mathrm{S}$ & 1,03 & 0,98 & & & \\
\hline Il vaut mieux être marié-e & $\overline{\mathrm{X}}$ & 4,55 & 4,57 & $-0,19$ & 1638 & non \\
\hline avant d'avoir des enfants & $\mathrm{S}$ & 1,56 & 1,51 & & & \\
\hline Dans notre société, il est & $\overline{\mathrm{x}}$ & 4,34 & 4,16 & 2,25 & 1626 & oui \\
\hline trop facile de divorcer & $\mathrm{S}$ & 1,56 & 1,64 & & & \\
\hline La relation entre les parents & $\overline{\mathrm{x}}$ & 3,30 & 3,60 & $-4,01$ & 1656,4 & oui \\
\hline $\begin{array}{l}\text { et les enfants est plus } \\
\text { importante que la relation } \\
\text { entre les parents eux-mêmes }\end{array}$ & $\mathrm{S}$ & 1,56 & 1,46 & & & \\
\hline La relation entre moi et & $\overline{\mathrm{x}}$ & 5,35 & 5,25 & 2,25 & 1603 & oui \\
\hline $\begin{array}{l}\text { mon ou ma conjoint-e } \\
\text { sera harmonieuse }\end{array}$ & $\mathrm{S}$ & 0,94 & 1,00 & & & \\
\hline Un jour, le destin fera que & $\overline{\mathrm{x}}$ & 4,55 & 4,25 & 3,90 & 1608 & oui \\
\hline $\begin{array}{l}\text { je rencontrerai l'homme } \\
\text { ou la femme de ma vie }\end{array}$ & $\mathrm{S}$ & 1,49 & 1,54 & & & \\
\hline
\end{tabular}


seconds $(5,06$ pour 4,79$)$; mais, pour les deux ensembles, le symbole du mariage est interpellant. Cette faible différence à la faveur des filles et cette vision se traduisent à nouveau quand les jeunes envisagent la possibilité de rencontrer l'homme ou la femme de leur vie (les moyennes sont alors de 4,55 et de 4,25 pour les filles et les garçons respectivement) et lorsqu'ils ont en tête la forme de la relation qu'ils entretiendront avec leur conjoint, car cette relation sera fort probablement heureuse (les moyennes étant de 5,35 et 5,25). Les filles hésitent un peu plus que les garçons à affirmer qu'il vaut mieux avoir un emploi stable avant de vivre en couple (les moyennes sont de 3,99 et 4,42) ; elles hésitent un peu moins à penser que divorcer est trop facile dans notre société (les moyennes sont de 4,34 pour elles et de 4,16 pour eux). Qu'ils soient de sexe masculin ou féminin, les jeunes témoignent d'une nette prudence devant une déclaration selon laquelle la relation entre les parents et les enfants est plus importante que celle qui a lieu entre les parents eux-mêmes ; mais la prudence des filles est un peu plus forte que celle des garçons (on obtient des moyennes de 3,30 pour celles-là et de 3,60 pour ceux-ci). Cette réserve n'empêche pas que les uns et les autres soient assez convaincus que leur relation entre chacun d'eux et leurs enfants sera harmonieuse, et les filles un peu plus que les garçons (on note des moyennes de 5,50 et de 5,41). Tous ces tests font état d'une différence entre les deux sexes, il est vrai. Mais aucune de ces comparaisons ne révèle d'écart marqué. La soustraction dont le résultat est le plus élevé est de 0,43 ; elle se rapporte à 1'opinion sur le préalable de l'emploi stable relativement à la vie de couple. Ainsi, malgré que ces inégalités soient inférables, on peut parler d'une ressemblance entre les deux sexes pour ce qui est de la façon dont on conçoit la famille.

La prise en considération des écarts types ne conduit pas à modifier ces constats. Ces mesures de dispersion sont normalement au-dessus de 1 sans jamais atteindre la valeur de 2 . Il y a donc quelques différences entre les individus pour ce qui est du niveau 
d'accord à l'égard des énoncés, mais ces variations n'atténuent pas vraiment les tendances centrales.

Le genre ne semble pas avoir de nette incidence sur la vision de la famille. Plusieurs positions sont claires, pour les filles et les garçons : les moyennes sont supérieures à 5 pour les deux sexes dans cinq cas. Le mariage, la rencontre de l'âme sœur constituent des symboliques actives. Avoir des enfants, c'est sérieux, et cela ne doit pas avoir lieu n'importe quand dans la vie.

\subsection{L'année d'études}

La façon de se représenter la famille varie-t-elle selon que l'élève est au début ou à la fin des études secondaires ?

À nouveau, pour répondre à cette question nous avons recouru aux tests $\mathrm{t}$ (voir le tableau 2). Ces tests ont été positifs six fois sur quatorze.

Les tests invitent donc à considérer les moyennes comme égales entre elles relativement à huit énoncés. Qu'on soit en neuvième année ou en douzième, les opinions sont du même ordre pour ce qui est des trois propositions qui se réfèrent au mariage, pour les deux qui posent les études comme antécédentes et pour les deux qui laissent espérer des relations harmonieuses.

L'accord est légèrement plus fort pour les élèves de neuvième année quand il est question de l'ascendant de la relation entre les parents et les enfants sur la relation entre les parents eux-mêmes, quand la phrase annonce la rencontre de l'homme ou la femme de sa vie et quand est exprimée l'idée d'une antériorité d'un emploi stable sur la vie de couple. L'assentiment est un peu plus élevé chez les élèves de douzième lorsqu'il a trait au rêve d'une vie amoureuse stable, à l'importance de faire précéder d'un emploi stable la venue des enfants et au jugement selon lequel il est trop facile de divorcer. Il semble donc que, en vieillissant, l'adolescent privilégie un peu plus le couple, mais diminue quelque peu ses attentes quant à l'éventuelle rencontre du partenaire idéal tout en entretenant, paradoxalement, un peu plus le rêve d'une vie amoureuse stable ; il apparaît aussi que sa position sur le divorce 
Tableau 2

Différence de moyennes pour diverses attitudes à l'égard de la famille selon l'année d'études

Tests $\mathrm{t}$

\begin{tabular}{|c|c|c|c|c|c|c|}
\hline$(1=$ pas du tou & $\mathrm{td}$ & ccord & $=$ tout à & t'acco & & \\
\hline Énoncé & & Ann & 'études & $\mathrm{t}$ & Ddl & $\mathrm{p}<0,05$ \\
\hline & & $9^{\mathrm{e}}$ & $12^{\mathrm{e}}$ & & & \\
\hline Plus tard, je me marierai & $\overline{\mathrm{X}}$ & 4,92 & 4,94 & $-0,17$ & 1617 & non \\
\hline & $\mathrm{s}$ & 1,32 & 1,34 & & & \\
\hline Plus tard, je vivrai avec un-e & $\overline{\mathrm{X}}$ & 2,35 & 2,33 & 0,16 & 1527 & non \\
\hline $\begin{array}{l}\text { conjoint-e, mais je ne me } \\
\text { marierai pas }\end{array}$ & $\mathrm{S}$ & 1,40 & 1,41 & & & \\
\hline Ma vie amoureuse sera stable & $\overline{\mathrm{x}}$ & 4,60 & 4,81 & $-3,27$ & 1609 & oui \\
\hline & $\mathrm{s}$ & 1,26 & 1,22 & & & \\
\hline Il vaut mieux avoir terminé ses & $\overline{\mathrm{x}}$ & 4,10 & 3,98 & 1,55 & 1661 & non \\
\hline études avant de vivre en couple & $\mathrm{s}$ & 1,55 & 1,62 & & & \\
\hline Il vaut mieux avoir terminé ses & $\overline{\mathrm{x}}$ & 5,19 & 5,30 & $-1,71$ & 1649,7 & non \\
\hline études avant d'avoir des enfants & $\mathrm{s}$ & 1,25 & 1,14 & & & \\
\hline Il vaut mieux avoir un emploi & $\overline{\mathrm{x}}$ & 4,36 & 4,02 & 4,73 & 1659 & oui \\
\hline stable avant de vivre en couple & $\mathrm{s}$ & 1,45 & 1,48 & & & \\
\hline Il vaut mieux avoir un emploi & $\overline{\mathrm{x}}$ & 5,26 & 5,41 & $-2,90$ & 1641,1 & oui \\
\hline stable avant d'avoir des enfants & $\mathrm{s}$ & 1,13 & 1,01 & & & \\
\hline La relation entre moi et mes & $\overline{\mathrm{x}}$ & 5,47 & 5,46 & 0,39 & 1584 & non \\
\hline enfants sera harmonieuse & $\mathrm{s}$ & 0,89 & 0,88 & & & \\
\hline La relation entre mon ou ma & $\overline{\mathrm{X}}$ & 5,33 & 5,37 & $-0,89$ & 1561 & non \\
\hline $\begin{array}{l}\text { conjoint-e et nos enfants sera } \\
\text { harmonieuse }\end{array}$ & $\mathrm{s}$ & 1,03 & 0,95 & & & \\
\hline Il vaut mieux être marié-e & $\overline{\mathrm{X}}$ & 4,55 & 4,57 & $-0,27$ & 1610 & non \\
\hline avant d'avoir des enfants & $\mathrm{S}$ & 1,55 & 1,53 & & & \\
\hline Dans notre société, il est trop & $\overline{\mathrm{X}}$ & 4,05 & 4,50 & $-5,68$ & 1601 & oui \\
\hline facile de divorcer & $\mathrm{S}$ & 1,61 & 1,55 & & & \\
\hline La relation entre les parents et & $\overline{\mathrm{x}}$ & 3,59 & 3,28 & 4,10 & 1644 & oui \\
\hline $\begin{array}{l}\text { les enfants est plus importante } \\
\text { que la relation entre les parents } \\
\text { eux-mêmes }\end{array}$ & $\mathrm{S}$ & 1,49 & 1,53 & & & \\
\hline La relation entre moi et mon ou & $\overline{\mathrm{x}}$ & 5,29 & 5,31 & $-0,29$ & 1576 & non \\
\hline ma conjoint-e sera harmonieuse & $\mathrm{s}$ & 1,00 & 0,93 & & & \\
\hline $\begin{array}{l}\text { Un jour, le destin fera que je } \\
\text { rencontrerai l'homme ou la } \\
\text { femme de ma vie }\end{array}$ & $\begin{array}{l}\bar{X} \\
S\end{array}$ & $\begin{array}{l}4,55 \\
1,47\end{array}$ & $\begin{array}{l}4,22 \\
1,58\end{array}$ & 4,20 & 1501,6 & oui \\
\hline
\end{tabular}


soit moins favorable et que s'accentue chez lui l'importance d'une bonne situation financière avant d'avoir des enfants.

Les différences de moyennes pour lesquelles on devrait croire qu'elles ne sont pas attribuables au hasard sont toujours faibles : elles vont de 0,45 (sur le divorce) à 0,15 (sur le rapport entre l'emploi stable et l'arrivée des enfants). Ainsi, bien qu'il faille admettre que les positions se modifient quelque peu du début à la fin de ces études secondaires, il faut aussi reconnaître que les opinions sur la famille sont plutôt stables. Les valeurs des écarts types confirment cette observation puisqu'elles sont toutes inférieures à 1,63 .

Dans l'ensemble, l'intensité des moyennes est la même que celle qui s'est manifestée quand les comparaisons ont porté sur le genre. Les moyennes, par exemple, sont au sommet quand allusion est faite à la relation entre soi et les enfants qu'on aura ; elles sont au plus bas quand l'énoncé indique que la vie de couple ne donnera pas lieu au mariage.

\subsection{La taille de la communauté de résidence}

Y a-t-il une différence dans l'imaginaire familial selon que la communauté dans laquelle on réside est grande ou petite?

C'est en recourant à des analyses de variance que nous avons répondu à cette question, car le facteur comporte quatre catégories (voir le tableau 3). Ces analyses ont détecté six cas d'inégalités de moyennes.

La taille de la municipalité dans laquelle les jeunes habitent ne fait pas varier le niveau d'assentiment sur le lien qui est fait entre la vie à deux et le mariage, sur la perspective d'une vie amoureuse stable, sur celle d'une relation stable avec le conjoint ni sur celle de la rencontre du partenaire désirable ; l'accord est encore le même quand sont évoqués le lien entre la fin des études et la vie de couple et celui entre un emploi stable et la vie de couple, quand sont distinguées la relation entre les parents et les enfants et la relation des parents entre eux, et lorsqu'il est fait mention de la trop grande aisance avec laquelle on recourt au divorce. 


\section{Tableau 3}

Différence de moyennes pour diverses attitudes à l'égard de la famille selon la taille de la communauté de résidence

Analyses de variance

\begin{tabular}{|c|c|c|c|c|c|c|}
\hline \multicolumn{7}{|c|}{$\begin{array}{l}\text { (Le degré de liberté minimum pour les individus est de } 1552) \\
(1=\text { pas du tout d'accord et } 6=\text { tout à fait d'accord })\end{array}$} \\
\hline & \multicolumn{4}{|c|}{ Taille de la communauté de résidence } & \multirow[t]{2}{*}{$\mathrm{F}$} & \multirow[t]{2}{*}{$\mathrm{p}<0,05$} \\
\hline & $<1000$ & $\begin{array}{c}\text { Entre } \\
1000 \text { et } \\
4999\end{array}$ & \begin{tabular}{|c|} 
Entre \\
5000 et \\
10999
\end{tabular} & Timmins & & \\
\hline \multirow[t]{2}{*}{ Plus tard, je me marierai } & 4,69 & 4,79 & 5,04 & 5,03 & \multirow[t]{2}{*}{7,42} & \multirow[t]{2}{*}{ oui } \\
\hline & 1,43 & 1,40 & 1,26 & 1,29 & & \\
\hline \multirow{2}{*}{$\begin{array}{l}\text { Plus tard, je vivrai avec un-e } \\
\text { conjoint-e, mais je ne me } \\
\text { marierai pas }\end{array}$} & 2,40 & 2,44 & 2,29 & 2,29 & \multirow[t]{2}{*}{0,91} & \multirow[t]{2}{*}{ non } \\
\hline & 1,44 & 1,49 & 1,36 & 1,40 & & \\
\hline \multirow[t]{2}{*}{ Ma vie amoureuse sera stable } & 4,58 & 4,60 & 4,76 & 4,77 & \multirow[t]{2}{*}{2,40} & \multirow[t]{2}{*}{ non } \\
\hline & 1,29 & 1,29 & 1,21 & 1,21 & & \\
\hline \multirow{2}{*}{$\begin{array}{l}\text { Il vaut mieux avoir terminé ses } \\
\text { études avant de vivre en couple }\end{array}$} & 3,87 & 4,09 & 4,03 & 4,18 & \multirow[t]{2}{*}{2,60} & \multirow[t]{2}{*}{ non } \\
\hline & 1,58 & 1,58 & 1,57 & 1,57 & & \\
\hline \multirow{2}{*}{$\begin{array}{l}\text { Il vaut mieux avoir terminé ses } \\
\text { études avant d'avoir des enfants }\end{array}$} & 5,04 & 5,13 & 5,29 & 5,39 & \multirow[t]{2}{*}{6,50} & \multirow[t]{2}{*}{ oui } \\
\hline & 1,37 & 1,24 & 1,14 & 1,02 & & \\
\hline \multirow{2}{*}{$\begin{array}{l}\text { Il vaut mieux avoir un emploi } \\
\text { stable avant de vivre en couple }\end{array}$} & 4,19 & 4,23 & 4,13 & 4,35 & \multirow[t]{2}{*}{1,92} & \multirow[t]{2}{*}{ non } \\
\hline & 1,50 & 1,44 & 1,44 & 1,49 & & \\
\hline \multirow{2}{*}{$\begin{array}{l}\text { Il vaut mieux avoir un emploi } \\
\text { stable avant d'avoir des enfants }\end{array}$} & 5,23 & 5,24 & 5,33 & 5,49 & \multirow[t]{2}{*}{4,61} & \multirow[t]{2}{*}{ oui } \\
\hline & 1,17 & 1,17 & 1,07 & 0,90 & & \\
\hline \multirow{2}{*}{$\begin{array}{l}\text { La relation entre moi et mes } \\
\text { enfants sera harmonieuse }\end{array}$} & 5,32 & 5,44 & 5,50 & 5,52 & \multirow[t]{2}{*}{4,04} & \multirow[t]{2}{*}{ oui } \\
\hline & 1,04 & 1,00 & 0,85 & 0,80 & & \\
\hline \multirow{2}{*}{$\begin{array}{l}\text { La relation entre mon ou ma } \\
\text { conjoint-e et nos enfants sera } \\
\text { harmonieuse }\end{array}$} & 5,25 & 5,28 & 5,33 & 5,50 & \multirow[t]{2}{*}{4,46} & oui \\
\hline & 1,09 & 1,11 & 1,01 & 0,80 & & \\
\hline Il vaut mieux être marié-e avant & 4,43 & 4,59 & 4,50 & 4,77 & 3,59 & oui \\
\hline d'avoir des enfants & 1,55 & 1,44 & 1,59 & 1,45 & & \\
\hline Dans notre société, il est trop & 4,09 & 4,36 & 4,22 & 4,37 & 2,41 & non \\
\hline facile de divorcer & 1,65 & 1,61 & 1,58 & 1,59 & & \\
\hline La relation entre les parents et les & 3,41 & 3,60 & 3,36 & 3,57 & 2,37 & non \\
\hline $\begin{array}{l}\text { enfants est plus importante que } \\
\text { la relation entre les parents } \\
\text { eux-mêmes }\end{array}$ & 1,57 & 1,50 & 1,51 & 1,48 & & \\
\hline La relation entre moi et mon ou & 5,23 & 5,23 & 5,30 & 5,41 & 2,61 & non \\
\hline ma conjoint-e sera harmonieuse & 1,08 & 1,04 & 0,93 & 0,87 & & \\
\hline Un jour, le destin fera que je & 4,32 & 4,35 & 4,47 & 4,38 & 0,88 & non \\
\hline $\begin{array}{l}\text { rencontrerai l'homme ou la } \\
\text { femme de ma vie }\end{array}$ & 1,53 & 1,56 & 1,49 & 1,55 & & \\
\hline
\end{tabular}


C'est dans les petites localités que le degré d'assentiment est le plus faible, et pour les six propositions qui donnent lieu à des différences inférables de moyennes, soit celle du projet de mariage, celle du rapport entre un emploi et l'arrivée des enfants, celle de l'importance d'avoir terminé ses études avant d'avoir des enfants, celle de la qualité de la relation entre soi et les enfants qu'on aura, celle de l'harmonie de la relation entre le conjoint et les enfants et celle du lien entre le mariage et le fait d'avoir des enfants. Dans cinq de ces six inégalités de moyennes, la valeur la plus élevée correspond à la ville de Timmins ; dans l'autre cas (« plus tard je me marierai »), la moyenne la plus forte est celle des municipalités qui comptent entre 5000 et 10999 habitants $(5,04)$, mais il faut la considérer comme égale à celle de Timmins $(5,03)$. Quand, donc, il y a inégalité de moyennes, la tendance veut que l'écart se situe entre la ville la plus peuplée et les localités les moins populeuses. L'optimisme et l'adhésion aux principes, quand il y a effectivement différence de moyennes, se veulent un peu plus nets à Timmins que dans les milieux les moins urbanisés. Il faut toutefois avoir à l'esprit que les soustractions entre le score le plus élevé et le plus faible ne se situent jamais au-delà de 0,54 , ce qui rappelle que, si les différences sont inférables, elles ne sont pas pour autant de grande amplitude. Encore une fois, les écarts types, lesquels sont toujours inférieurs à deux, rappellent que les opinions sont plutôt concentriques.

\subsection{La langue}

L'image qu'on a de la famille diffère-t-elle selon la langue maternelle?

Ce sont les analyses de variance qui ont permis de répondre à cette question puisque le facteur comprend trois modalités (voir le tableau 4). Cette fois, ce sont neuf tests qui repèrent des inégalités de moyennes.

La langue maternelle n'a pas d'incidence sur le niveau d'accord pour cinq énoncés. Le premier est celui du mariage dans la vie de couple; le deuxième parle du rapport entre la vie 
de couple et la fin des études; le troisième a trait au lien entre un emploi stable et le fait d'avoir des enfants; le quatrième se rapporte à l'harmonie de la relation entre soi et les enfants; le cinquième distingue la relation entre les parents et les enfants de la relation entre les parents eux-mêmes.

Dans les neuf cas d'inégalités de moyennes, les jeunes qui déclarent à la fois le français et l'anglais comme langues maternelles se situent toujours entre les francophones et les anglophones. La moyenne des francophones est supérieure à celle des anglophones pour sept propositions : «plus tard je me marierai », « ma vie amoureuse sera stable », « il vaut mieux avoir terminé ses études avant d'avoir des enfants », « la relation entre mon ou ma conjoint-e et nos enfants sera harmonieuse », « dans notre société, il est trop facile de divorcer », « la relation entre moi et mon ou ma conjoint-e sera harmonieuse » et « un jour, le destin fera que je rencontrerai l'homme ou la femme de ma vie ». La moyenne des anglophones est la plus élevée relativement à deux énoncés : « il vaut mieux avoir un emploi stable avant de vivre en couple » et « il vaut mieux être marié-e avant d'avoir des enfants ». La différence de moyennes est au plus fort quand il s'agit de la rencontre éventuelle du partenaire de vie ; elle est de 0,55 . L'écart est à son point le plus faible quand la proposition fait référence à la qualité de la relation entre soi et les enfants. Les positions des francophones et des anglophones tendent donc vers une certaine similarité quoiqu'il faille constater que les principes et les idéaux sont légèrement plus définis dans l'imaginaire des francophones que dans celui des anglophones. Si l'on tient compte des écarts types, on n'a pas à moduler ces remarques.

Les moyennes sont fortement corrélées en fonction des énoncés avec celles qu'on découvre dans les analyses où l'on compare les sexes ou les niveaux de scolarité : quand elles sont fortes ailleurs, elles le sont aussi ici.

Cette réponse à la question de l'incidence de la langue maternelle ayant été donnée, il est permis de se demander si une analyse en fonction de la langue de l'école que fréquentent 
Tableau 4

Différence de moyennes pour diverses attitudes à l'égard de la famille selon la-les langue-s maternelle-s

Analyses de variance

\begin{tabular}{|c|c|c|c|c|c|c|}
\hline $\begin{array}{l}\text { (Le degré de libert } \\
(1=\text { pas du to }\end{array}$ & & $\begin{array}{l}\text { imum pou } \\
\text { ccord et } 6\end{array}$ & $\begin{array}{l}r \text { les indi } \\
=\text { tout à }\end{array}$ & $\begin{array}{l}\text { vidus est de } \\
\text { fait d'accor }\end{array}$ & 1493) & \\
\hline Énoncé & & Langu & e-s mater & nelle-s & $\mathrm{F}$ & $\mathrm{p}<0,05$ \\
\hline & & Français & Anglais & $\begin{array}{l}\text { Français } \\
\text { et anglais }\end{array}$ & & \\
\hline Plus tard, je me marierai & $\overline{\mathrm{X}}$ & 5,08 & 4,81 & 5,03 & 7,06 & oui \\
\hline & $\mathrm{S}$ & 1,25 & 1,37 & 1,26 & & \\
\hline Plus tard, je vivrai avec un-e & $\overline{\mathrm{X}}$ & 2,35 & 2,27 & 2,37 & 0,79 & non \\
\hline $\begin{array}{l}\text { conjoint-e, mais je ne me } \\
\text { marierai pas }\end{array}$ & $\mathrm{S}$ & 1,41 & 1,40 & 1,41 & & \\
\hline Ma vie amoureuse sera stable & $\overline{\mathrm{X}}$ & 4,98 & 4,55 & 4,72 & 13,84 & oui \\
\hline & $\mathrm{S}$ & 1,17 & 1,29 & 1,20 & & \\
\hline Il vaut mieux avoir terminé ses & $\bar{x}$ & 3,99 & 4,05 & 4,09 & 0,47 & non \\
\hline études avant de vivre en couple & $\mathrm{S}$ & 1,65 & 1,51 & 1,59 & & \\
\hline Il vaut mieux avoir terminé ses & $\bar{x}$ & 5,41 & 5,11 & 5,34 & 9,89 & oui \\
\hline études avant d'avoir des enfants & $\mathrm{S}$ & 1,13 & 1,23 & 1,15 & & \\
\hline Il vaut mieux avoir un emploi & $\overline{\mathrm{X}}$ & 3,95 & 4,35 & 4,21 & 8,84 & oui \\
\hline stable avant de vivre en couple & $\mathrm{S}$ & 1,50 & 1,41 & 1,46 & & \\
\hline Il vaut mieux avoir un emploi & $\overline{\mathrm{X}}$ & 5,23 & 5,36 & 5,38 & 2,37 & non \\
\hline stable avant d'avoir des enfants & $\mathrm{s}$ & 1,20 & 1,04 & 0,99 & & \\
\hline La relation entre moi et mes & $\overline{\mathrm{X}}$ & 5,48 & 5,41 & 5,53 & 2,78 & non \\
\hline enfants sera harmonieuse & $\mathrm{S}$ & 0,82 & 1,00 & 0,76 & & \\
\hline La relation entre mon ou ma & $\bar{x}$ & 5,45 & 5,26 & 5,39 & 5,04 & oui \\
\hline $\begin{array}{l}\text { conjoint-e et nos enfants sera } \\
\text { harmonieuse }\end{array}$ & $\mathrm{s}$ & 0,87 & 1,10 & 0,94 & & \\
\hline Il vaut mieux être marié-e avant & $\overline{\mathrm{X}}$ & 4,32 & 4,74 & 4,51 & 9,10 & oui \\
\hline d'avoir des enfants & $\mathrm{S}$ & 1,66 & 1,44 & 1,53 & & \\
\hline Dans notre société, il est trop & $\bar{x}$ & 4,46 & 4,12 & 4,26 & 5,01 & oui \\
\hline facile de divorcer & $\mathrm{S}$ & 1,53 & 1,62 & 1,63 & & \\
\hline La relation entre les parents et & $\overline{\mathrm{X}}$ & 3,34 & 3,48 & 3,46 & 1,00 & non \\
\hline $\begin{array}{l}\text { les enfants est plus importante } \\
\text { que la relation entre les parents } \\
\text { eux-mêmes }\end{array}$ & $\mathrm{s}$ & 1,58 & 1,48 & 1,52 & & \\
\hline La relation entre moi et mon ou & $\overline{\mathrm{X}}$ & 5,44 & 5,19 & 5,35 & 8,59 & oui \\
\hline ma conjoint-e sera harmonieuse & $\mathrm{s}$ & 0,87 & 1,07 & 0,89 & & \\
\hline Un jour, le destin fera que je & $\overline{\mathrm{X}}$ & 4,71 & 4,16 & 4,51 & 16,95 & oui \\
\hline $\begin{array}{l}\text { rencontrerai l'homme ou la } \\
\text { femme de ma vie }\end{array}$ & $\mathrm{s}$ & 1,32 & 1,61 & 1,48 & & \\
\hline
\end{tabular}


les élèves fournirait des résultats concordants. On perdrait alors la modalité « français et anglais », puisqu'il n'y a pas d'école bilingue au sens strict. La comparaison ne porterait que sur deux langues : « française » et « anglaise » (voir le tableau 5). La question a quelque pertinence puisqu' on peut s'imaginer que ce resserrement définirait mieux les deux groupes linguistiques, mettant en évidence une espèce de traditionalisme qu'on entreverrait volontiers plus intense chez les francophones que chez les anglophones. Si l'on effectuait cette analyse, dix tests produiraient les mêmes résultats que dans l'examen précédent. Parmi les quatre autres, trois, de négatifs qu'ils étaient, deviendraient positifs; ils se rapporteraient aux énoncés suivants : « ma vie amoureuse sera stable », « il vaut mieux avoir un emploi stable avant d'avoir des enfants » et « la relation entre les parents et les enfants est plus importante que la relation entre les parents eux-mêmes ». Dans les trois cas, bien que la différence deviendrait inférable par l'élimination d'une modalité, la différence serait minime : 0,18, - 0,20 et - 0,26. En vertu d'une proposition, le test deviendrait négatif : « la relation entre mon ou ma conjoint-e et nos enfants sera harmonieuse »; il faudrait donc considérer comme équivalentes les moyennes pour l'anglais et le français. Pour le premier énoncé qui deviendrait positif, celui qui donne à entrevoir le concubinage plutôt que le mariage, le désaccord serait un peu plus prononcé chez les élèves de langue anglaise $(2,29)$ que chez ceux qui sont de langue française $(2,47)$, la désapprobation se rapprochant de la valeur 1. Avec le deuxième, celui qui note la préférence de détenir un emploi avant d'avoir des enfants, l'assentiment serait de 5,38 pour les écoles anglaises et de 5,18 pour les françaises. Le troisième, qui parle d'un ascendant de la relation entre les parents sur la relation entre les parents et les enfants, signalerait aussi un accord légèrement supérieur des élèves anglais $(3,52)$ sur les français $(3,26)$. On ne pourrait certainement pas conclure à une mise en relief d'un conformisme francophone. Devant toutes les nouvelles moyennes et tous les nouveaux écarts types, ce qui frapperait le plus, ce 
Tableau 5

Différence de moyennes pour diverses attitudes à l'égard de la famille selon qu'il s'agit d'une école de langue française ou de langue anglaise

Tests $\mathrm{t}$

\begin{tabular}{|c|c|c|c|c|c|c|}
\hline \multicolumn{7}{|c|}{$(1=$ pas du tout d'accord et $6=$ tout à fait d'accord $)$} \\
\hline \multirow[t]{2}{*}{ Énoncé } & & \multicolumn{2}{|c|}{ Langue de l'école } & \multirow[t]{2}{*}{$\mathrm{t}$} & \multirow[t]{2}{*}{$\mathrm{ddl}$} & \multirow[t]{2}{*}{$\mathrm{p}<0,05$} \\
\hline & & Française & Anglaise & & & \\
\hline \multirow[t]{2}{*}{ Plus tard, je me marierai } & $\overline{\mathrm{X}}$ & 5,09 & 4,87 & \multirow[t]{2}{*}{3,10} & \multirow[t]{2}{*}{814,19} & \multirow[t]{2}{*}{ oui } \\
\hline & $\mathrm{s}$ & 1,24 & 1,36 & & & \\
\hline \multirow{2}{*}{$\begin{array}{l}\text { Plus tard, je vivrai avec } \\
\text { un-e conjoint-e, mais je } \\
\text { ne me marierai pas }\end{array}$} & $\bar{X}$ & 2,47 & 2,29 & \multirow[t]{2}{*}{2,21} & \multirow[t]{2}{*}{1554} & \multirow[t]{2}{*}{ oui } \\
\hline & $\mathrm{s}$ & 1,48 & 1,39 & & & \\
\hline \multirow[t]{2}{*}{ Ma vie amoureuse sera stable } & $\overline{\mathrm{x}}$ & 4,99 & 4,59 & \multirow[t]{2}{*}{6,02} & \multirow[t]{2}{*}{858,42} & \multirow[t]{2}{*}{ oui } \\
\hline & $\mathrm{s}$ & 1,13 & 1,27 & & & \\
\hline \multirow{2}{*}{$\begin{array}{l}\text { Il vaut mieux avoir terminé } \\
\text { ses études avant de vivre } \\
\text { en couple }\end{array}$} & $\overline{\mathrm{x}}$ & 3,96 & 4,06 & \multirow[t]{2}{*}{$-1,13$} & \multirow[t]{2}{*}{719,70} & \multirow[t]{2}{*}{ non } \\
\hline & $\mathrm{s}$ & 1,69 & 1,54 & & & \\
\hline \multirow{2}{*}{$\begin{array}{l}\text { Il vaut mieux avoir terminé } \\
\text { ses études avant d'avoir } \\
\text { des enfants }\end{array}$} & $\overline{\mathrm{X}}$ & 5,43 & 5,16 & \multirow[t]{2}{*}{4,29} & \multirow[t]{2}{*}{879,46} & \multirow[t]{2}{*}{ oui } \\
\hline & $\mathrm{s}$ & 1,10 & 1,24 & & & \\
\hline \multirow{2}{*}{$\begin{array}{l}\text { Il vaut mieux avoir un emploi } \\
\text { stable avant de vivre en couple }\end{array}$} & $\overline{\mathrm{x}}$ & 3,90 & 4,31 & \multirow[t]{2}{*}{$-5,13$} & \multirow[t]{2}{*}{1690} & \multirow[t]{2}{*}{ oui } \\
\hline & $\mathrm{s}$ & 1,51 & 1,43 & & & \\
\hline & $\overline{\mathrm{x}}$ & 5,18 & 5,38 & $-3,20$ & 693,83 & oui \\
\hline stable avant d'avoir des enfants & $\mathrm{s}$ & 1,19 & 1,03 & & & \\
\hline La relation entre moi et mes & $\overline{\mathrm{x}}$ & 5,43 & 5,47 & $-0,69$ & 1613 & non \\
\hline enfants sera harmonieuse & $\mathrm{s}$ & 0,83 & 0,93 & & & \\
\hline La relation entre mon ou ma & $\overline{\mathrm{x}}$ & 5,38 & 5,33 & 0,91 & 1588 & non \\
\hline $\begin{array}{l}\text { conjoint-e et nos enfants } \\
\text { sera harmonieuse }\end{array}$ & $\mathrm{S}$ & 0,96 & 1,02 & & & \\
\hline Il vaut mieux être marié-e & $\overline{\mathrm{x}}$ & 4,25 & 4,67 & $-4,71$ & 697,81 & oui \\
\hline avant d'avoir des enfants & $\mathrm{S}$ & 1,66 & 1,47 & & & \\
\hline Dans notre société, il est trop & $\overline{\mathrm{X}}$ & 4,39 & 4,19 & 2,25 & 1628 & oui \\
\hline facile de divorcer & $\mathrm{s}$ & 1,56 & 1,62 & & & \\
\hline La relation entre les parents et & $\overline{\mathrm{X}}$ & 3,26 & 3,52 & $-3,11$ & 1675 & oui \\
\hline $\begin{array}{l}\text { les enfants est plus importante } \\
\text { que la relation entre les parents } \\
\text { eux-mêmes }\end{array}$ & $\mathrm{S}$ & 1,56 & 1,49 & & & \\
\hline La relation entre moi et mon & $\overline{\mathrm{X}}$ & 5,41 & 5,26 & 3,13 & 975,94 & oui \\
\hline $\begin{array}{l}\text { ou ma conjoint-e sera } \\
\text { harmonieuse }\end{array}$ & $\mathrm{s}$ & 0,80 & 1,03 & & & \\
\hline Un jour, le destin fera que je & $\bar{x}$ & 4,84 & 4,24 & 7,84 & 935,80 & oui \\
\hline $\begin{array}{l}\text { rencontrerai l'homme ou la } \\
\text { femme de ma vie }\end{array}$ & $\mathrm{s}$ & 1,27 & 1,58 & & & \\
\hline
\end{tabular}


serait la proximité des chiffres par comparaison aux précédents. Il faudrait donc soutenir que les valeurs que l'école transmet sont largement celles que les groupes linguistiques véhiculent, ce qui ne devrait pas étonner. Par ailleurs, l'analogie sur la base de la langue de l'école, en augmentant de deux le nombre de tests positifs, accentuerait quelque peu la différence interlinguistique, mais elle ne le ferait jamais dans une grande mesure. Ainsi, si l'école se rapporte à des différences linguistiquement sensibles, elle traduit aussi ce que la société, d'une façon générale, fait de l'ensemble de ses citoyens, indépendamment de leur langue.

\subsection{La religion}

La religion fait-elle fluctuer la manière dont on se représente l'amour et la vie familiale?

Nous ne disposons pas d'un fort indicateur de la religion. Nous ne sommes pas en mesure de déterminer jusqu'à quel point un élève a ou non la foi, est ou non pratiquant. Nous savons par contre si les jeunes fréquentent des écoles catholiques ou non confessionnelles. Si l'on veut démolir cette variable, on peut faire valoir que tous les jeunes qui étudient dans les écoles catholiques ne sont pas issus de foyers catholiques ou même que tous ceux qui sont inscrits dans les écoles publiques ne sont pas athées. Mais on n'arrivera pas à démontrer que la variable est nulle. Il est tout de même fortement probable que des parents catholiques envoient leur enfant dans une école catholique, comme est forte aussi la probabilité que des parents non catholiques inscrivent leur enfant dans une école non confessionnelle. Mais ce n'est que selon cette logique que la variable est utile.

Les tests sont alors neuf fois positifs et cinq fois négatifs (voir le tableau 6). Parmi les tests positifs, la moyenne est supérieure chez les catholiques pour six énoncés : «plus tard, je me marierai », « il vaut mieux avoir terminé ses études avant d'avoir des enfants », « la relation entre mon et ma conjoint-e et nos enfants sera harmonieuse », " dans notre société, il est trop facile de divorcer », « la relation entre moi et mon ou ma conjoint-e 
Tableau 6

Différence de moyennes pour diverses attitudes à l'égard de la famille selon que l'école est catholique ou publique

Tests $\mathrm{t}$

\begin{tabular}{|c|c|c|c|c|c|c|}
\hline \multicolumn{7}{|c|}{$(1=$ pas du tout d'accord et $6=$ tout à fait d'accord $)$} \\
\hline \multirow[t]{2}{*}{ Énoncé } & & \multicolumn{2}{|c|}{ École } & \multirow[t]{2}{*}{$\mathrm{t}$} & \multirow[t]{2}{*}{ ddl } & \multirow[t]{2}{*}{$\mathrm{p}<0,05$} \\
\hline & & publique & catholique & & & \\
\hline \multirow[t]{2}{*}{ Plus tard, je me marierai } & $\overline{\mathrm{X}}$ & 4,83 & 5,04 & \multirow[t]{2}{*}{$-3,21$} & \multirow[t]{2}{*}{1626,50} & \multirow[t]{2}{*}{ oui } \\
\hline & $\mathrm{s}$ & 1,36 & 1,29 & & & \\
\hline \multirow{2}{*}{$\begin{array}{l}\text { Plus tard, je vivrai avec un-e } \\
\text { conjoint-e, mais je ne me } \\
\text { marierai pas }\end{array}$} & $\overline{\mathrm{X}}$ & 2,28 & 2,41 & \multirow[t]{2}{*}{$-1,85$} & \multirow[t]{2}{*}{1554} & \multirow[t]{2}{*}{ non } \\
\hline & $\mathrm{S}$ & 1,38 & 1,45 & & & \\
\hline \multirow[t]{2}{*}{ Ma vie amoureuse sera stable } & $\overline{\mathrm{X}}$ & 4,52 & 4,90 & \multirow[t]{2}{*}{$-6,32$} & \multirow[t]{2}{*}{1631,22} & \multirow[t]{2}{*}{ oui } \\
\hline & $\mathrm{S}$ & 1,28 & 1,17 & & & \\
\hline \multirow{2}{*}{$\begin{array}{l}\text { Il vaut mieux avoir terminé } \\
\text { ses études avant de vivre } \\
\text { en couple }\end{array}$} & $\overline{\mathrm{X}}$ & 4,02 & 4,05 & \multirow[t]{2}{*}{$-0,41$} & \multirow[t]{2}{*}{1595,67} & \multirow[t]{2}{*}{ non } \\
\hline & $\mathrm{S}$ & 1,50 & 1,67 & & & \\
\hline \multirow{2}{*}{$\begin{array}{l}\text { Il vaut mieux avoir terminé ses } \\
\text { études avant d'avoir des enfants }\end{array}$} & $\overline{\mathrm{x}}$ & 5,07 & 5,42 & \multirow[t]{2}{*}{$-5,99$} & \multirow[t]{2}{*}{1687,94} & \multirow[t]{2}{*}{ oui } \\
\hline & $\mathrm{s}$ & 1,27 & 1,11 & & & \\
\hline \multirow{2}{*}{$\begin{array}{l}\text { Il vaut mieux avoir un emploi } \\
\text { stable avant de vivre en couple }\end{array}$} & $\overline{\mathrm{X}}$ & 4,30 & 4,10 & \multirow[t]{2}{*}{2,85} & \multirow[t]{2}{*}{1690} & \multirow[t]{2}{*}{ oui } \\
\hline & $\mathrm{S}$ & 1,41 & 1,52 & & & \\
\hline Il vaut mieux avoir un emploi & $\bar{x}$ & 5,36 & 5,29 & 1,36 & 1673 & non \\
\hline stable avant d'avoir des enfants & $\mathrm{S}$ & 1,06 & 1,10 & & & \\
\hline La relation entre moi et mes & $\bar{x}$ & 5,45 & 5,46 & $-0,13$ & 1613 & non \\
\hline enfants sera harmonieuse & $\mathrm{S}$ & 0,95 & 0,85 & & & \\
\hline La relation entre mon ou ma & $\overline{\mathrm{x}}$ & 5,27 & 5,43 & $-3,08$ & 1587,99 & oui \\
\hline $\begin{array}{l}\text { conjoint-e et nos enfants } \\
\text { sera harmonieuse }\end{array}$ & $\mathrm{S}$ & 1,07 & 0,92 & & & \\
\hline Il vaut mieux être marié-e & $\overline{\mathrm{x}}$ & 4,69 & 4,40 & 3,76 & 1533,35 & oui \\
\hline avant d'avoir des enfants & $\mathrm{S}$ & 1,45 & 1,61 & & & \\
\hline Dans notre société, il est & $\overline{\mathrm{x}}$ & 4,11 & 4,40 & $-3,71$ & 1628 & oui \\
\hline trop facile de divorcer & $\mathrm{S}$ & 1,63 & 1,57 & & & \\
\hline La relation entre les parents et & $\overline{\mathrm{x}}$ & 3,47 & 3,44 & 0,30 & 1620,20 & non \\
\hline $\begin{array}{l}\text { les enfants est plus importante } \\
\text { que la relation entre les } \\
\text { parents eux-mêmes }\end{array}$ & $\mathrm{S}$ & 1,48 & 1,56 & & & \\
\hline La relation entre moi et mon & $\overline{\mathrm{x}}$ & 5,20 & 5,42 & $-4,65$ & 1595,47 & oui \\
\hline $\begin{array}{l}\text { ou ma conjoint-e sera } \\
\text { harmonieuse }\end{array}$ & $\mathrm{S}$ & 1,06 & 0,85 & & & \\
\hline Un jour, le destin fera que je & $\overline{\mathrm{x}}$ & 4,15 & 4,67 & $-6,98$ & 1606,64 & oui \\
\hline $\begin{array}{l}\text { rencontrerai l'homme ou la } \\
\text { femme de ma vie }\end{array}$ & $\mathrm{S}$ & 1,62 & 1,36 & & & \\
\hline
\end{tabular}


sera harmonieuse » et « un jour, le destin fera que je rencontrerai l'homme ou la femme de ma vie ». La différence la plus faible $(+0,11)$ se rapporte à l'assertion « plus tard, je me marierai »; la plus forte $(+0,52)$, à celle qui veut qu' « un jour, le destin fera que je rencontrerai l'homme ou la femme de ma vie ». Ainsi, l'écart entre les deux ensembles n'est jamais prononcé et il est plus marqué à l'égard d'idéaux amoureux que de consécration de la vie de couple. La moyenne est plus élevée dans les écoles publiques à l'égard de trois énoncés « ma vie amoureuse sera stable », « il vaut mieux avoir un emploi stable avant de vivre en couple », « il vaut mieux être marié-e avant d'avoir des enfants ». Les différences sont faibles à nouveau : elles se situent entre 0,20 et 0,38 . Pour les cinq autres, il faut considérer les moyennes comme égales. On ne peut assurément pas affirmer, à la lumière de ces informations, que le mariage est catholique : les moyennes qui ont trait aux trois énoncés qui font allusion à cette institution rappellent que les deux groupes sont proches l'un de l'autre et les deux cas d'inégalité indiquent un plus grand accord une fois dans les écoles catholiques («plus tard je me marierai »), l'autre fois, dans les écoles publiques « il vaut mieux être marié-e avant d'avoir des enfants ». À nouveau, on est tenu de relever une grande similitude entre les groupes, assortie de quelques particularités.

\subsection{L'interaction des facteurs}

Est-ce que les facteurs interviennent de façon interactive sur les opinions à l'égard de la famille ?

Pour le découvrir, nous avons testé les effets d'interaction pour les six facteurs pris deux à deux sur tous les énoncés. Sur les 210 tests qui ont ainsi été effectués, sept se sont avérés positifs. On pourrait être tenté de déclarer ces résultats comme étant tout simplement des effets du hasard, car ce hasard peut effectivement faire que sept tests sur 210 se révèlent comme inférables. Et c'est bien ce que nous aurions fait si, sur ces sept tests, les résultats avaient semblé aléatoires. Or tel n'est pas le cas, car les variables année d'études et langue maternelle se manifestent chacune 
quatre fois, et celle de la taille de la communauté de résidence, trois fois ; de surcroît, deux variables dépendantes sont deux fois l'enjeu de ces interactions : « la relation entre les parents et les enfants est plus importante que la relation entre les parents euxmêmes » et « la relation entre moi et mon ou ma conjoint-e sera harmonieuse ». Aussi nous semble-t-il plus prudent d'examiner ces effets d'interaction que de les écarter d'entrée de jeu par peur de commettre une erreur de première espèce.

La taille de la communauté de résidence interagit deux fois avec l'année d'études et une fois avec la langue maternelle. Les moyennes sont d'abord celles qui sont relatives à la vision d'une relation heureuse entre le conjoint et les enfants (voir le tableau 7). À s'en remettre aux tests d'hypothèse, il semble que la différence de moyennes selon la population ne dépende pas de l'année d'études - puisqu'on lit « oui » deux fois - mais que la différence entre les années d'études dépende, elle, de la taille de la communauté. Les moyennes sont considérées comme inégales aux niveaux neuf et douze; elles le sont dans les communautés de moins de 1000 habitants et dans celles qui comptent entre 1000 et 4999 citoyens. En deçà de ces tests, il faut noter que la moyenne maximale aux niveaux neuf et douze est celle de Timmins $(5,48$ et 5,53 respectivement) mais que la plus faible, en neuvième, est celle des communautés dans lesquelles on dénombre entre 1000 et 4999 individus $(5,17)$ alors que, en douzième, c'est celle des plus petites localités $(5,10)$. Ainsi, on note que l'opinion sur la perspective de la relation entre le conjoint et les enfants est la même, indépendamment du niveau d'études, dans les milieux de résidences les plus populeux, mais qu'elle varie dans les autres de telle sorte que c'est en neuvième qu'elle est la plus optimiste dans les milieux de moins de 1000 habitants $(5,41)$ et que cet optimisme, en douzième, est plus grand dans les lieux où il y a entre 1000 et 4999 citoyens $(5,44)$. 


\section{Tableau 7}

Décomposition de l'effet d'interaction des variables taille de la communauté et année de scolarité pour l'énoncé « la relation entre mon ou ma conjoint-e et nos enfants sera harmonieuse »

\begin{tabular}{|c|c|c|c|c|c|}
\hline \multicolumn{6}{|c|}{$\begin{array}{c}\mathrm{F}_{(3 ; 1555)}=5,09 ; \mathrm{p}<0,01 \\
\text { (Moyenne et écart type et test à } \mathrm{p}<0,05) \\
(1=\text { tout à fait d'accord et } 6=\text { pas du tout d'accord })\end{array}$} \\
\hline & & \multirow{2}{*}{$\mathrm{p}<0,05$} \\
\hline & $\begin{array}{c}\text { Moins de } \\
1000\end{array}$ & $\begin{array}{c}\text { Entre } 1000 \\
\text { et } 4999\end{array}$ & $\begin{array}{c}\text { Entre } 5000 \\
\text { et } 10999\end{array}$ & Timmins & \\
\hline $9^{\mathrm{e}}$ & $\begin{array}{c}5,41 \\
(0,92)\end{array}$ & $\begin{array}{c}5,17 \\
(1,24)\end{array}$ & $\begin{array}{c}5,27 \\
(1,06)\end{array}$ & $\begin{array}{c}5,48 \\
(0,87)\end{array}$ & oui \\
\hline $12^{\mathrm{e}}$ & $\begin{array}{c}5,10 \\
(1,19) \\
\end{array}$ & $\begin{array}{c}5,44 \\
(0,88) \\
\end{array}$ & $\begin{array}{c}5,41 \\
(0,92) \\
\end{array}$ & $\begin{array}{c}5,53 \\
(0,70) \\
\end{array}$ & oui \\
\hline $\mathrm{p}<0,05$ & oui & oui & non & non & \\
\hline
\end{tabular}

Les moyennes pour lesquelles on trouve des effets d'interaction qui impliquent la population de la communauté se rapportent ensuite à l'image du bonheur entre le conjoint et soi. Une première fois, le facteur se combine à celui de l'année d'études (voir le tableau 8). Les tests inférentiels font dépendre aussi bien de l'année d'études l'incidence de la taille de la communauté (un test est positif, l'autre, non) que de la taille de la communauté celle de l'année d'étude (trois tests sont négatifs, l'autre, non). Quand on est en neuvième année, la perspective d'une harmonie conjugale ne dépend pas de la taille de la communauté ; quand on est en douzième, dans les plus petites communautés $(5,06)$, la moyenne est plus faible qu'ailleurs (entre 5,31 et 5,44 ) ; elle est aussi plus faible, dans ces petites localités lorsqu'on est en douzième année que lorsqu'on est en neuvième $(5,39)$.

En vertu du même énoncé, la taille de la communauté entretient un lien aussi avec la langue maternelle (voir le tableau 9). L'effet de la taille de la localité dépend aussi bien de langue maternelle (un « oui » et deux « non ») que l'effet de la langue maternelle de la localité (deux « oui » et deux « non »). Les moyennes qui sont calculées en fonction de la population du lieu d'habitation ne sont inégales que chez les individus dont la langue maternelle 
Tableau 8

Décomposition de l'effet d'interaction des variables taille de la communauté et année de scolarité pour l'énoncé

« la relation entre moi et mon ou ma conjoint-e sera harmonieuse »

\begin{tabular}{|c|c|c|c|c|c|}
\hline \multicolumn{6}{|c|}{$\begin{array}{c}\mathrm{F}_{(3 ; 1570)}=5,00 ; \mathrm{p}<0,01 \\
(\text { Moyenne et écart type et test à } \mathrm{p}<0,05) \\
(1=\text { tout à fait d'accord et } 6=\text { pas du tout d'accord })\end{array}$} \\
\hline \multirow[t]{2}{*}{ Année scolaire } & \multicolumn{4}{|c|}{ Population de la communauté de résidence } & \multirow[t]{2}{*}{$\mathrm{p}<0,05$} \\
\hline & $\begin{array}{c}\text { Moins de } \\
1000\end{array}$ & $\begin{array}{c}\text { Entre } 1000 \\
\text { et } 4999\end{array}$ & $\begin{array}{c}\text { Entre } 5000 \\
\text { et } 10999\end{array}$ & Timmins & \\
\hline $9^{\mathrm{e}}$ & $\begin{array}{c}5,39 \\
(0,99)\end{array}$ & $\begin{array}{c}5,37 \\
(1,14)\end{array}$ & $\begin{array}{c}5,26 \\
(0,96)\end{array}$ & $\begin{array}{c}5,38 \\
(0,95)\end{array}$ & non \\
\hline $12^{\mathrm{e}}$ & $\begin{array}{c}5,06 \\
(1,12)\end{array}$ & $\begin{array}{c}5,44 \\
(0,87)\end{array}$ & $\begin{array}{c}5,34 \\
(0,90)\end{array}$ & $\begin{array}{c}5,31 \\
(0,93)\end{array}$ & oui \\
\hline $\mathrm{p}<0,05$ & oui & non & non & non & \\
\hline
\end{tabular}

\section{Tableau 9}

Décomposition de l'effet d'interaction

des variables taille de la communauté et langue-s maternelle-s pour l'énoncé

« la relation entre moi et mon ou ma conjoint-e sera harmonieuse »

\begin{tabular}{|c|c|c|c|c|c|}
\hline & $\begin{array}{r}\text { (Moy } \\
=\text { tout à }\end{array}$ & $\begin{array}{l}; 1532)=2,51 \\
\text { et écart type } \\
\text { accord et } 6\end{array}$ & $\begin{array}{l}<0,01 \\
\text { test à } \mathrm{p}<0 \\
\text { pas du tout }\end{array}$ & ) & \\
\hline Langue-s & & on de la com & nauté de résic & & $p<0,05$ \\
\hline maternelle-s & $\begin{array}{c}\text { Moins de } \\
1000\end{array}$ & $\begin{array}{c}\text { Entre } 1000 \\
\text { et } 4999\end{array}$ & $\begin{array}{c}\text { Entre } 5000 \\
\text { et } 10999\end{array}$ & Timmins & \\
\hline Français & $\begin{array}{c}5,40 \\
(0,94)\end{array}$ & $\begin{array}{c}5,40 \\
(0,74)\end{array}$ & $\begin{array}{c}5,44 \\
(0,87)\end{array}$ & $\begin{array}{c}5,49 \\
(0,88)\end{array}$ & non \\
\hline Anglais & $\begin{array}{c}5,08 \\
(1,18)\end{array}$ & $\begin{array}{c}5,00 \\
(1,23)\end{array}$ & $\begin{array}{c}5,25 \\
(1,00)\end{array}$ & $\begin{array}{c}5,40 \\
(0,79)\end{array}$ & oui \\
\hline $\begin{array}{l}\text { Français et } \\
\text { anglais }\end{array}$ & $\begin{array}{c}5,35 \\
(0,98)\end{array}$ & $\begin{array}{c}5,59 \\
(0,55)\end{array}$ & $\begin{array}{c}5,25 \\
(0,89)\end{array}$ & $\begin{array}{c}5,41 \\
(0,95)\end{array}$ & non \\
\hline $\mathrm{p}<0,05$ & oui & oui & non & non & \\
\hline
\end{tabular}

est l'anglais ; on observe que, dans les environnements les plus peuplés, les moyennes sont un peu plus fortes $(5,25$ et 5,40$)$ que dans les lieux qui le sont moins $(5,00$ et 5,08$)$. Les moyennes, par ailleurs, ne diffèrent pas d'après la langue maternelle dans les deux premières catégories de municipalités, mais elles le font dans les deux autres. Là où il y a moins de 1000 individus, la 
moyenne est plus faible chez les anglophones $(5,08)$ que chez les francophones $(5,40)$ ou chez les bilingues de naissance $(5,35)$ et il en va de même dans les milieux qui comptent entre 1000 et 4999 citoyens (où les chiffres sont respectivement de 5,00, 5,40 et 5,59). Ainsi, c'est chez les jeunes de langue maternelle anglaise et dans les localités de moins de 5000 habitants que la perspective d'une relation harmonieuse avec le conjoint est la moins optimiste.

Aucune de toutes ces moyennes n'est inférieure à cinq, ce qui donne à observer un optimisme étendu aussi bien en ce qui a trait à la relation entre le conjoint et les enfants qu'entre le conjoint et soi. Cependant, on doit noter que, dans les milieux où il y a au moins 5000 habitants, les moyennes sont toujours égales et que, dans les plus petites communautés, il y a des variations en fonction de l'année d'études et de la langue maternelle, l'optimisme étant moins marqué chez les élèves de douzième que chez ceux de neuvième et moins prononcé aussi chez les jeunes dont la langue maternelle est l'anglais.

La langue maternelle interagit avec la taille de la communauté de résidence, nous venons de le constater. Elle le fait aussi avec le niveau d'études, la confessionnalité du système d'éducation et le sexe. Le premier objet de ces interactions est l'énoncé selon lequel la vie amoureuse sera stable (voir le tableau 10). Les tests a posteriori révèlent que les moyennes sont inégales entre les catégories de langue maternelle aussi bien pour les élèves de neuvième que pour ceux de douzième mais que, entre les élèves de neuvième et ceux de douzième, il n'y a d'inégalité que chez les jeunes qui déclarent deux langues maternelles. Il y a donc différence de moyennes en fonction de la langue, qu'on soit en neuvième ou en douzième ; on peut ajouter que, chez les élèves de neuvième comme chez ceux de douzième, la confiance est plus élevée chez les francophones (les moyennes étant de 4,91 et 5,06 respectivement) que chez les anglophones (dans le même ordre 4,54 et 4,56). Ni chez les francophones, ni chez les anglophones les moyennes ne changent de la neuvième à la douzième année ; 
Tableau 10

Décomposition de l'effet d'interaction des variables langue-s maternelle-s et année de scolarité

pour l'énoncé

« ma vie amoureuse sera stable »

\begin{tabular}{|c|c|c|c|c|}
\hline \multicolumn{5}{|c|}{$\begin{array}{c}\mathrm{F}_{(2 ; 1545)}=3,37 ; \mathrm{p}<0,05 \\
(\text { Moyenne et écart type et test à } \mathrm{p}<0,05) \\
(1=\text { tout à fait d'accord et } 6=\text { pas du tout d'accord })\end{array}$} \\
\hline \multirow[t]{2}{*}{ Année scolaire } & \multicolumn{3}{|c|}{$\begin{array}{l}\text { Langue-s maternelle-s } \\
\end{array}$} & \multirow[t]{2}{*}{$\mathrm{p}<0,05$} \\
\hline & Français & Anglais & Français et anglais & \\
\hline $9^{e}$ & $\begin{array}{c}4,91 \\
(1,14)\end{array}$ & $\begin{array}{c}4,54 \\
(1,30)\end{array}$ & $\begin{array}{c}4,56 \\
(1,25)\end{array}$ & oui \\
\hline $12^{\mathrm{e}}$ & $\begin{array}{c}5,06 \\
(1,20)\end{array}$ & $\begin{array}{c}4,56 \\
(1,29)\end{array}$ & $\begin{array}{c}4,94 \\
(1,10)\end{array}$ & oui \\
\hline $\mathrm{p}<0,05$ & non & non & oui & \\
\hline
\end{tabular}

on note une faible inégalité chez les jeunes qui se disent bilingues de naissance (la moyenne passant de 4,56 en neuvième à 4,94 en douzième).

La langue maternelle agit conjointement avec le système scolaire selon qu'il est catholique ou public et les moyennes sont calculées pour la proposition « la relation entre moi et mes enfants sera harmonieuse » (voir le tableau 11). D'après les tests inférentiels, l'interaction est double : les variations déterminées par la langue dépendent du système scolaire (on a un « oui » et un « non ») et celles qui sont attribuables au système scolaire dépendent de la langue maternelle (on a deux « non » et un « oui »). Chez les élèves du système public, la moyenne la plus forte est celle des bilingues $(5,65)$; chez ceux du système catholique, cette moyenne est celle des anglophones $(5,53)$. Les moyennes doivent être considérées comme égales entre elles, que les élèves soient de langue maternelle française ou anglaise, entre le système public et le système catholique ; il y a toutefois une faible différence entre les deux systèmes chez les jeunes qui se déclarent bilingues de naissance. 


\section{Tableau 11}

Décomposition de l'effet d'interaction

des variables langue-s maternelle-s et système scolaire

pour l'énoncé

« la relation entre moi et mes enfants sera harmonieuse »

\begin{tabular}{|l|c|c|c|c|}
\hline \multicolumn{5}{|c|}{$\mathrm{F}_{(2 ; 1543)}=4,15 ; \mathrm{p}<0,05$} \\
$($ Moyenne et écart type et test à p $<0,05)$ \\
$(1=$ tout à fait d'accord et 6 $=$ pas du tout d'accord) \\
\cline { 2 - 4 } Système scolaire & \multicolumn{3}{|c|}{ Langue-s maternelle-s } & \multirow{2}{*}{$<0,05$} \\
& Français & Anglais & Français et anglais & \\
\hline Public & 5,23 & 5,39 & 5,65 & oui \\
& $(1,31)$ & $(1,02)$ & $(0,62)$ & \\
\hline Catholique & 5,49 & 5,53 & 5,44 & non \\
& $(0,78)$ & $(0,87)$ & $(0,85)$ & \\
\hline $\mathrm{p}<0,05$ & non & non & oui & \\
\hline
\end{tabular}

La langue maternelle produit un effet combiné, enfin, avec le sexe (voir le tableau 12) et il concerne l'assertion d'après laquelle la relation entre les parents et les enfants aurait préséance sur celle qu'il y a entre les parents. L'analyse montre que l'influence de la langue maternelle dépend du sexe de l'élève (on note un « oui » et un «non ») tout aussi bien que l'effet du sexe sur l'opinion dépend de la langue maternelle (on note deux « oui » et un « non ») : il n’y a pas de différence de moyennes chez les garçons entre les groupes linguistiques, mais il y en a une chez les filles, les filles de langue maternelle française $(3,08)$ croyant moins que les anglophones $(3,26)$ et que les bilingues de naissance $(3,47)$ que la relation entre les parents et les enfants doive être prioritaire ; il n'y a pas de différence de moyennes, chez les jeunes qui sont bilingues de naissance, entre les filles et les garçons, mais il y a de telles différences chez les francophones et chez les anglophones, dans les deux cas l'assentiment étant plus faible chez les filles.

Quand on invite les élèves à imaginer leur relation amoureuse et celle qu'ils auront avec leurs enfants, les francophones et les anglophones sont comparables, sans considération pour l'année d'études et pour le système d'éducation, quoiqu'il faille signaler 


\section{Tableau 12}

Décomposition de l'effet d'interaction

des variables langue-s maternelle-s et sexe

pour l'énoncé

« la relation entre les parents et les enfants est plus importante

que la relation entre les parents eux-mêmes »

\begin{tabular}{|c|c|c|c|c|}
\hline \multicolumn{5}{|c|}{$\begin{array}{c}\mathrm{F}_{(2 ; 1604)}=4,37 ; \mathrm{p}<0,05 \\
(\text { Moyenne et écart type et test à } \mathrm{p}<0,05) \\
(1=\text { tout à fait d'accord et } 6=\text { pas du tout d'accord })\end{array}$} \\
\hline \multirow[t]{2}{*}{ Sexe } & \multicolumn{3}{|c|}{ Langue-s maternelle-s } & \multirow[t]{2}{*}{$\mathrm{p}<0,05$} \\
\hline & Français & Anglais & Français et anglais & \\
\hline Féminin & $\begin{array}{c}3,08 \\
(1,58)\end{array}$ & $\begin{array}{c}3,26 \\
(1,55)\end{array}$ & $\begin{array}{c}3,47 \\
(1,53) \\
\end{array}$ & oui \\
\hline Masculin & $\begin{array}{c}3,59 \\
(1,54)\end{array}$ & $\begin{array}{c}3,67 \\
(1,39)\end{array}$ & $\begin{array}{c}3,46 \\
(1,50)\end{array}$ & non \\
\hline $\mathrm{p}<0,05$ & oui & oui & non & \\
\hline
\end{tabular}

de légères différences chez les jeunes qui se disent de langue maternelle à la fois française et anglaise. Devant un principe qui veut que la relation entre les parents et les enfants soit plus importante que celle des parents entre eux, c'est chez les francophones et chez les anglophones qu'il y a une différence de moyennes, cette fois entre les sexes, les filles étant plus hésitantes que les garçons. Entre les groupes linguistiques, on remarque une plus grande confiance quant à la stabilité amoureuse chez les francophones, quel que soit le niveau d'études, de plus grands espoirs à l'endroit de la relation avec les enfants chez les bilingues dans le système scolaire public et un accord plus prononcé chez les filles bilingues que chez les autres. Jamais, les différences de moyennes, toutefois, ne sont fortes.

La septième interaction a trait une deuxième fois à l'ascendant du rapport entre les parents et les enfants sur celui entre les parents eux-mêmes et les deux facteurs qui interagissent sont le niveau d'études et le sexe (voir le tableau 13). On remarque qu'il n'y a pas de différence entre les niveaux d'études chez les élèves de sexe masculin mais qu'il y en a une chez ceux qui sont de sexe féminin, les filles de douzième $(3,03)$ étant encore plus réticentes à admettre le propos que celles de neuvième $(3,54)$. Quand ils 


\section{Tableau 13}

Décomposition de l'effet d'interaction des variables année de scolarité et sexe pour l'énoncé

« la relation entre les parents et les enfants est plus importante que la relation entre les parents eux-mêmes »

\begin{tabular}{|c|c|c|c|}
\hline \multicolumn{4}{|c|}{$\begin{array}{c}\mathrm{F}_{(1 ; 1640)}=8,23 ; \mathrm{p}<0,05 \\
\text { (Moyenne et écart type et test à } \mathrm{p}<0,05) \\
(1=\text { tout à fait d'accord et } 6=\text { pas du tout d'accord })\end{array}$} \\
\hline \multirow[t]{2}{*}{ Sexe } & \multicolumn{2}{|c|}{ Année scolaire } & \multirow[t]{2}{*}{$\mathrm{p}<0,05$} \\
\hline & $9^{\mathrm{e}}$ & $12^{\mathrm{e}}$ & \\
\hline Féminin & $\begin{array}{c}3,54 \\
(1,52)\end{array}$ & $\begin{array}{c}3,03 \\
(1,55)\end{array}$ & oui \\
\hline Masculin & $\begin{array}{c}3,63 \\
(1,46)\end{array}$ & $\begin{array}{c}3,54 \\
(1,47)\end{array}$ & non \\
\hline $\mathrm{p}<0,05$ & non & oui & \\
\hline
\end{tabular}

sont en neuvième année, les élèves ont des opinions équivalentes s'ils sont de sexe masculin ou de sexe féminin ; entre les garçons et les filles, il y a toutefois une différence si les élèves sont en douzième année, l'hésitation étant un peu plus forte chez les filles $(3,03)$ que chez les garçons $(3,54)$.

\subsection{Des opinions plutôt nettes, des nuances et des propos généralisés}

Si l'on exclut les analyses sur les effets d'interaction, nous avons effectué quatre-vingt-quatre tests sur les différences de moyennes, soit une série de quatorze tests pour chacun des six facteurs. Au total, un peu plus de la moitié de ces tests se sont avérés positifs $(48 / 84)$. Aucun d'entre eux n'a fait état de différence supérieure à 0,60 (sur des échelles à six niveaux). On a donc affaire à une thématique sur laquelle les opinions sont plutôt semblables quoiqu'elles fassent place à des différences relatives.

Il y a une assertion pour laquelle les moyennes sont constamment égales : « il vaut mieux avoir terminé ses études avant de vivre en couple $»$.

Il y a une seule proposition qui ne subit l'effet que d'un seul facteur : « plus tard, je vivrai avec un-e conjoint-e, mais je ne 
me marierai pas » et un seul également qui ne réagisse que par rapport à deux facteurs : " la relation entre moi et mes enfants sera harmonieuse $»$.

Il y a trois énoncés dont les moyennes varient relativement à trois facteurs : « il vaut mieux avoir un emploi stable avant d'avoir des enfants », « la relation entre mon ou ma conjoint-e et nos enfants sera harmonieuse » et « la relation entre les parents et les enfants est plus importante que la relation entre les parents eux-mêmes ».

Il y en a quatre dont les inégalités sont attribuables à quatre facteurs : « ma vie amoureuse sera stable », « il vaut mieux avoir terminé ses études avant d'avoir des enfants », « il vaut mieux être marié-e avant d'avoir des enfants » et « la relation entre moi et mon ou ma conjoint-e sera harmonieuse ».

Il y en a quatre aussi qui fluctuent d'après cinq facteurs : « plus tard, je me marierai », « il vaut mieux avoir un emploi stable avant de vivre en couple », « dans notre société, il est trop facile de divorcer » et « un jour, le destin fera que je rencontrerai l'homme ou la femme de ma vie ».

Il n'y a pas de structure nette à la source de ces variations. On peut remarquer que l'année d'études et la taille de la communauté interviennent six fois, le sexe, sept fois, la langue maternelle et le système d'éducation neuf fois, la langue de l'école, enfin, onze fois. La langue maternelle et la langue de l'école influent huit fois sur les mêmes variables et, chaque fois, le système scolaire a aussi une influence, trois fois et le sexe et l'année d'études ont aussi leur effet. C'est donc dire que trois fois, les moyennes varient en fonction de cinq facteurs semblables ; c'est le cas pour les énoncés « il vaut mieux avoir un emploi stable avant de vivre en couple », " dans notre société, il est trop facile de divorcer » et « un jour le destin fera que je rencontrerai l'homme ou la femme de ma vie ». La proximité entre la langue de l'école et la langue maternelle ne saurait étonner ; il faut signaler la parenté, également, entre la langue de l'école et le caractère confessionnel de cette école, l'effet de la langue ressemblant souvent à celui 
du système scolaire, les catholiques se comportant souvent comme les francophones, ce qui ne fait que traduire le fait que les francophones s'inscrivent souvent dans les écoles catholiques.

Dans l'ensemble, les opinions sont assez nettes : si l'on excepte la proposition sur la prépondérance de la relation entre les parents et les enfants sur la relation entre les parents eux-mêmes, où les moyennes témoignent d'une hésitation (elles se situent entre trois et quatre), les moyennes sont au-dessus de quatre et, pour cinq énoncés, elles sont généralement supérieures à cinq ; elles sont inférieures à trois quand elles ont trait à une vie à deux qui exclut le mariage, ce qui témoigne toujours d'une position relativement claire.

Les effets d'interaction n'entravent pas cette tendance à s'exprimer plutôt nettement ; ils n'invalident pas l'influence des facteurs quand ils sont pris isolément. Ils rappellent l'importance de faire des nuances, ils soulignent que l'influence de la taille de la communauté de résidence et celle de la langue maternelle ne sont pas insensibles à d'autres facteurs comme l'année d'études, le sexe et la religion.

\section{Conclusion}

Les analyses qui ont été effectuées avaient pour but de répondre à diverses questions et de vérifier quelques hypothèses.

La première de ces questions avait trait aux perspectives amoureuses et conjugales des jeunes. Les statistiques montrent clairement que les jeunes imaginent une relation harmonieuse avec leur conjoint (les moyennes sont supérieures à cinq), mais elles signalent en même temps la présence d'un doute quant à la rencontre de « l'homme ou la femme de ma vie » (les moyennes sont au-dessus de quatre, mais à bonne distance de cinq) et à la stabilité de cette future vie (les moyennes n'atteignent pas la valeur de cinq, mais elles sont bien au-dessus de quatre). Le mariage interpelle beaucoup quoiqu'il laisse percevoir quelques hésitations (les moyennes sont plus près de cinq que de six); si l'énoncé a trait au concubinage qui serait préférable au mariage, 
le désaccord est dominant sans être absolu (les moyennes sont loin de 1 , mais elles sont tout de même supérieures à 2). Dans toutes ces projections, on peut sentir un certain effet de la fragilité des relations matrimoniales dont sont témoins les jeunes, mais cet effet n'est pas tel qu'il écarte le mariage des projets ou qu'il empêche de rêver à des amours harmonieuses. Sur certains de ces objets, on aperçoit un optimisme un peu plus prononcé chez les filles que chez les garçons, mais on remarque surtout une grande similitude des unes et des autres. On constate aussi que les opinions des plus jeunes sont comparables à celles des plus vieux, si ce n'est que ceux-ci croient légèrement plus à la stabilité amoureuse et que ceux-là espèrent un peu plus la rencontre de l'âme sœur. On note que, derrière une grande ressemblance des francophones et des anglophones, se profilent malgré tout des attentes plus confiantes des premiers et que, sur un solide fond de similarité, les jeunes tendent un peu moins à donner foi au mariage dans les petites communautés. On remarque que le caractère confessionnel ou non de l'école a peu d'effet, que s'il est vrai que les moyennes dans les écoles catholiques sont parfois supérieures à celles des écoles publiques sur des thématiques dont on pourrait dire qu'elles sont révélatrices d'un traditionalisme, d'une façon générale, les propos qui sont tenus dans ces écoles-là sont proches de ceux qui sont exprimées dans les écoles publiques, et à l'égard de certains propos traditionnels, les moyennes sont plus fortes dans les écoles publiques. L'hypothèse de l'idéalisme des jeunes filles est globalement fausse, comme l'est celle de la sagacité des élèves les plus vieux. L'hypothèse de l'équivalence des francophones et des anglophones est essentiellement vraie, mais on peut s'étonner de quelques espérances discrètement plus prononcées chez les francophones, comme si l'état de minoritaire invitait à quelque confiance supplémentaire. L'hypothèse de l'homogénéité relative aux communautés n'est pas vraiment contestable. L'hypothèse, enfin, d'un plus grand conservatisme des catholiques, dans la mesure où la confessionnalité de l'école est indicatrice de cette 
religiosité, n'est vraie que sur quelques objets et, quand elle l'est, ce n'est que marginalement.

La deuxième question concernait la relation entre le jeune et les enfants qu'il aura. Sur ce point, les attentes sont fortes (les moyennes sont au-delà de cinq). Il y a bien quelques décimales à la faveur des filles et des plus grandes communautés, mais rien qui puisse nier le fait généralisé de perspectives heureuses.

La troisième question portait sur l'éventuelle relation entre le conjoint et les enfants. Les moyennes sont un peu plus faibles que ne le sont celles qui ont trait à la relation entre soi et les enfants, mais elles sont tout de même fortes (supérieures à cinq), et elles ne varient, et faiblement, qu'en fonction de la taille de la communauté de résidence.

Ainsi les jeunes considèrent-ils que leur rôle de parent se jouera sous le signe de la bonne entente et qu'il en ira pareillement de celui qu'est appelé à jouer la personne avec laquelle ils auront ces enfants. Il semble donc que la précarité des familles nucléaires dont ils font le constat dans leur environnement n'interdise pas aux jeunes de croire en la concorde future des parents et des enfants.

Les jeunes se sont par ailleurs prononcés sur quelques propositions dont le contenu révélait un certain traditionalisme. Les analyses ont montré que s'ils sont fortement d'accord avec le principe de l'antériorité des études sur le fait d'avoir des enfants (les moyennes sont supérieures à cinq), ils le sont moins avec celui de la préséance des études sur la vie de couple, que, dans le même esprit, s'ils trouvent souhaitable d'avoir un emploi stable avant d'avoir des enfants (les moyennes dépassent la valeur de cinq), cet emploi est moins requis pour former un couple. Le mariage leur apparaît comme un préalable à la procréation, mais de façon relative (les moyennes se situent à mi-chemin entre quatre et cinq). Le divorce n'est pas a priori condamnable, mais leur accord à l'égard d'un énoncé qui veut qu'il soit trop facile de divorcer est assez marqué (les moyennes sont supérieures à quatre). Enfin, les jeunes expriment quelque indécision devant une 
assertion qui veut que la relation entre les parents et les enfants soit plus importante que celle qui implique les parents entre eux; on sent bien qu'ils voudraient répondre oui et non (les moyennes sont au beau milieu de l'échelle, entre trois et quatre). Sur tous ces sujets, les variations entre les sexes, les âges, les langues et les communautés sont négligeables.

Tous ces résultats révèlent la prégnance des modes traditionnels en même temps que l'ouverture à des événements contingents ; ils mettent en évidence des imaginaires animés par le rêve à la périphérie duquel pointe quelque crainte ; ils dépeignent une jeunesse troublée par l'incertitude matrimoniale et familiale, mais qui, malgré tout, parvient à entretenir des idéaux sur l'amour et sur les rapports entre les parents et les enfants.

Entre les francophones et les anglophones, il y a régulièrement des différences de moyennes, et ces inégalités sont parfois précisées par un facteur autre que la langue. On remarque alors que chacun de ces deux groupes linguistiques a des traits qui lui sont propres. Mais il y a surtout de grandes similitudes que signalent les égalités de moyennes et que souligne la faiblesse des inégalités. On note alors que ces deux populations appartiennent à une société qui livre à tous ses membres des caractéristiques communes. Il y a certainement des façons respectives aux francophones et aux anglophones d'envisager l'amour et de concevoir la famille, mais il y a aussi des modes sociétaux de le faire, des modes qui rendent irréductibles à des déterminants spécifiques des façons de faire et de penser. Dans cette étude, comme dans les précédentes - celles qui ont porté sur les médias, sur les aspirations éducationnelles et professionnelles et sur le passage de l'élémentaire au secondaire-, nous avons repéré une spécificité francophone, mais nous avons nettement été conduit à constater que le francophone et l'anglophone ont beaucoup en partage, qu'ils participent d'une société qui assure leur particularité mais qui, simultanément, ne les rend jamais tout à fait dissemblables. Sur une thématique comme celle des projets amoureux et familiaux, c'est sans doute à travers cette similitude que se dessinent les mariages hybrides ; mais c'est 
aussi dans le contexte des particularismes que se développent plusieurs conflits, dans ces mariages croisés, entre autres sur la langue, celle qui est employée entre les conjoints, celle dans laquelle on communique avec les enfants, celle dans laquelle on fait instruire les enfants, celle dont on fait usage pour échanger avec les membres de la famille élargie et avec les amis. Car une langue n'est jamais un simple moyen de communication; si elle n'est pas, en elle-même, l'intégralité d'une culture, elle est plus qu'un instrument qui sert à faire circuler de l'information. 